\title{
TILINGS OF THE TORUS AND THE KLEIN BOTTLE AND VERTEX-TRANSITIVE GRAPHS ON A FIXED SURFACE
}

\author{
CARSTEN THOMASSEN
}

\begin{abstract}
We describe all regular tilings of the torus and the Klein bottle. We apply this to describe, for each orientable (respectively nonorientable) surface $S$, all (but finitely many) vertex-transitive graphs which can be drawn on $S$ but not on any surface of smaller genus (respectively crosscap number). In particular, we prove the conjecture of Babai that, for each $g \geq 3$, there are only finitely many vertex-transitive graphs of genus $g$. In fact, they all have order $<10^{10} \mathrm{~g}$. The weaker conjecture for Cayley graphs was made by Gross and Tucker and extends Hurwitz' theorem that, for each $g \geq 2$, there are only finitely many groups that act on the surface of genus $g$. We also derive a nonorientable version of Hurwitz' theorem.
\end{abstract}

\section{INTRODUCTION}

Let $S_{g}$ and $N_{k}$ denote the orientable (respectively nonorientable) surface of genus $g$ (respectively crosscap number $k$ ). The genus (respectively crosscap number) of a graph $G$ is the smallest number $g$ (respectively $k$ ) such that $G$ can be embedded into $S_{g}$ (respectively $N_{k}$ ) such that the edges are pairwise noncrossing simple curves. By Hurwitz' theorem and examples by Wormald (see [4, p. 304]), the double torus $S_{2}$ has the following remarkable property: There are only finitely many groups that act on $S_{2}$ but there are infinitely many Cayley graphs of genus 2. Gross and Tucker [4] conjectured that no other orientable surface has this property. Babai (see [4]) made the stronger conjecture that, for each $g \geq 3$, there are only finitely many vertex-transitive graphs of genus $g$. The result described in the abstract proves this conjecture. In addition, for each $k \geq 4$, there are only finitely many vertex-transitive graphs of crosscap number $k$. (This will not be proved formally in the paper, though. We merely point out that our proof of Babai's conjecture extends to the nonorientable case as well.) Babai [13] has independently verified his conjecture by different methods. The only vertex-transitive graphs of genus 2 are (with a finite number of exceptions) graphs similar to the aforementioned examples of Wormald. All these graphs have natural embeddings in the Klein bottle. An inspection of our list of vertextransitive graphs shows that all (but finitely many) have unique embeddings and that their automorphisms can be extended to homeomorphisms of the surface.

Received by the editors September 6, 1988.

1980 Mathematics Subject Classification (1985 Revision). Primary 05C10, 57M15, 57S25. 
Using the results of this paper it will probably not be difficult to produce a complete catalogue of the vertex-transitive graphs of genus $g \leq 1$ (maybe even $g=2$ ) or crosscap number $k \leq 2$. For $g=0$ (the planar case) this was done by Fleischner and Imrich [3].

Tucker [11] proved that, if a finite group acts on a surface $S$ (that is, the group may be thought of as a group of homeomorphisms of $S$ ), then some Cayley graph $G$ of the group has a 2-cell embedding on $S$ such that every homeomorphism in the group induces an isomorphism (in fact, a left multiplication) of the graph. The results of the present paper tell us what the graph $G$ is, provided the group (and hence also $G$ ) is large. Thus we obtain an alternative description of all (but finitely many) finite homeomorphism groups of $S$. In particular, it follows by inspection of the list of graphs in this paper that $S_{0}, S_{1}, N_{1}, N_{2}$ are the only surfaces having infinitely many homeomorphism groups.

The main strategy in the paper is the following: We first describe completely all tilings of the torus $S_{1}$ and the Klein bottle $N_{2}$ into hexagons, quadrilaterals, and triangles in which the vertices have degree 3,4 , and 6 , respectively. (Part of this was also done by Altschuler [1].) We impose some natural regularity conditions on the tilings in order to avoid the degenerate ones. Many of these tilings are vertex-transitive graphs, and we describe some operations which will transform these graphs into new vertex-transitive graphs on the torus or the Klein bottle. We then show that these are the only (except finitely many) vertextransitive graphs on the torus or the Klein bottle. Finally, we show that every vertex-transitive graph which has many vertices compared to its genus, can be embedded in the torus or the Klein bottle, and, by inspection of our catalogue, we conclude that those on the Klein bottle (fortunately) can be embedded on the double torus. (Note, that this does not hold for all graphs on the Klein bottle; see $[4,8]$.) The following two observations (which will be made precise later) are important for the proof: First, Euler's formula implies that a large graph on a given surface has a vertex of degree at most 6 . Second, a large vertex-transitive graph $G$ of a given genus cannot contain a small nonplanar graph $H$. For if that were the case, then $G$ would contain many disjoint copies of $H$ (by the vertex-transitivity). But then $G$ would have large genus because of the additivity property of the graph genus [2], a contradiction.

All the vertex-transitive graphs described in this appear have Hamiltonian cycles. This supports the well-known conjecture of $L$. Lovász that all connected vertex-transitive graphs have a Hamiltonian path. For each fixed genus, the conjecture now is a finite problem.

The tilings considered in this paper are (locally) similar to some of the tilings of the plane studied by Grünbaum and Shephard [5]. However, it is not clear if there is a direct connection between the results on tilings in the present paper and those in [5]. Finally we point out that Babai's conjecture cannot be extended to graphs which are almost vertex-transitive in the sense that the vertex set has only two orbits under the automorphism group. If $G$ is a connected 1-transitive 
graph with $n$ vertices and $q$ edges, then we may form a new graph $G_{k}$ by replacing each edge $x y$ of $G$ by a $k$-cycle joined completely to $x$ and $y$. Clearly, $G_{k}$ has only two orbits under the automorphism group of $G_{k}$. It is shown in [9] that, for $k$ sufficiently large, $G_{k}$ has genus $q-n+1$.

\section{PRELIMINARY RESULTS ON SYMMETRIES AND EMBEDDINGS}

In this section we describe the graphs that will occur in the results of this paper and we prove some auxiliary results. First we repeat some standard definitions.

A graph $G$ is a pair $V(G)$ and $E(G)$, where $V(G)$ is a finite set (of vertices) and $E(G)$ is a set of unordered pairs $x y$ of vertices called edges. We say that the edge $x y$ joins $x$ and $y$ and that it incident with $x$ and $y$ and that $x$ and $y$ are neighbors. The order of $G$ is the cardinality of $V(G)$. The $G$-degree (or just degree) of the vertex $x$ is the number of neighbors of $x$ in $G$. The set of neighbors of $x$ is denoted $N(x)$ and we put $\bar{N}(x)=N(x) \cup\{x\}$. If $A \subseteq V(G) \cup E(G)$, then $G-A$ is the graph obtained from $G$ by deleting $A$ and all edges incident with $A \cap V(G)$. If $A \subseteq V(G)$, then the graph $G(A)$ induced by $A$ is defined as $G-(V(G) \backslash A)$. The graph induced by $N(x)$ (respectively $\bar{N}(x)$ ) is the neighborhood graph (respectively closed neighborhood graph) of $x$. A graph is $d$-regular if all vertices have degree $d$. A 3-regular graph is also called cubic. A $d$-factor of $G$ is a $d$-regular subgraph containing all vertices of $G$. A $k$-path is a graph with vertices $x_{0}, x_{1}, \ldots, x_{k}$ and edges $x_{i-1} x_{i}$, $i=1,2, \ldots, k$. A $k$-cycle is obtained from a $(k-1)$-path by adding the edge between the two ends (i.e., vertices of degree 1). We say that $k$ is the length of the $k$-path and $k$-cycle. A graph is connected if any two vertices are joined by a path. The distance between two vertices is the length of a shortest path between them. The eccentricity of a vertex $x$ is the maximum distance from $x$ to other vertices. The radius of a connected graph is the smallest eccentricity. A vertex whose eccentricity equals the radius is called a center of the graph. The girth of a graph is the length of a shortest cycle. A cycle through all vertices of $G$ is a Hamiltonian cycle of $G$. A graph $G$ is bipartite, if there is a partition $V(G)=A \cup B$ such that all edges of $G$ join $A$ and $B$. The complete graph $K_{n}$ is the graph of order $n$ where all possible edges are present.

We shall make use of some graph operations. If $C: x_{1} x_{2} \cdots x_{k} x_{1}$ is a $k$ cycle, then adding a new vertex $v$ and joining it to all vertices of $C$ results in a $k$-wheel with center $v$. If $k$ is even, then an edge of the type $x_{i} x_{k / 2+i}$ is called a diagonal of $C$.

The square $G^{2}$ of a graph $G$ is obtained from $G$ by adding all edges between vertices of distance 2 in $G$. The line graph $L(G)$ of $G$ is the graph whose vertex set is $E(G)$ such that two vertices are neighbors in $L(G)$ iff they have a common end in $G$. The Cartesian product of two graphs $G$ and $H$ is the graph with vertex set $V(G) \times V(H)$ such that $(x, u)$ and $(y, v)$ are neighbors 
iff either $x=y$ and $u$ and $v$ are neighbors in $H$, or $u=v$ and $x$ and $y$ are neighbors in $G$.

If $x y$ is an edge of $G$, then contracting $x y$ means that we delete the edge $x y$ and identify $x$ and $y$. More generally, we may contract a number of edges in $G$. This will result in a graph which may contain multiple edges. Such a (generalized) graph is called a multigraph. If we replace every multiple edge by a single edge we speak of the underlying graph.

An automorphism of a graph $G$ is a mapping $\pi: V(G) \rightarrow V(G)$ which is 1-1 and onto such that, for any two vertices $x, y, x$ and $y$ are neighbors iff $\pi(x)$ and $\pi(y)$ are neighbors. The automorphism group of $G$ partitions $V(G)$ and $E(G)$ into orbits. $G$ is vertex-transitive (respectively edge-transitive) if $V(G)$ (respectively $E(G)$ ) has only one orbit. If, for any edges $x y, u v$, there is an automorphism taking $x$ to $u$ and $y$ to $v$ (and $G$ has no isolated vertex), then $G$ is 1 -transitive. Clearly, 1-transitivity implies vertex-transitivity and edgetransitivity. The converse is not true. Also, edge-transitivity does not imply vertex-transitivity. However, the following is an easy exercise (see, e.g., [12]; (c) below is an immediate consequence of (a) and (b)).

Lemma 2.1. (a) If $G$ is edge-transitive and has no isolated vertex, then either $G$ is vertex-transitive, or $V(G)$ has precisely two orbits and all edges of $G$ join vertices in distinct orbits. (In other words, $G$ is bipartite.)

(b) If $G$ is vertex-transitive, edge-transitive, and $d$-regular, where $d$ is odd, then $G$ is 1-transitive.

(c) If $G$ is edge-transitive, nonbipartite, and d-regular, where $d$ is odd, then $G$ is 1-transitive.

A surface is a connected compact topological Hausdorff space which is locally homeomorphic to a disc. By the classification theorem for surfaces (see, e.g., [4, 10]) the only surfaces are $S_{g}$, the sphere with $g$ handles, and $N_{k}$, the sphere with $k$ crosscaps. If $G$ is a graph drawn on a surface such that the edges are pairwise simple noncrossing curves, then $G$ partitions $S_{g}$ or $N_{k}$ into regions, called faces. If each face is homeomorphic to a disc, then the embedding is called a 2 -cell embedding. The genus $g(G)$ (respectively crosscap number $c(G)$ ) is the smallest number $m$ such that $G$ has an embedding in $S_{m}$ (respectively $N_{m}$ ). An embedding of $G$ into $S_{g(G)}$ is always a 2-cell embedding (see [4]).

Assume now that a connected graph $G$ is a 2-cell embedded in a surface $S$, where $S=S_{g}$ or $S=N_{k}$. The boundary of a face is called a facial walk. If the number of faces is denoted by $f$, and $|V(G)|=n$ and $|E(G)|=q$, then Euler's formula says that $n-q+f$ equals $2-2 g$ (when $S=S_{g}$ ) or $2-k$ (when $S=N_{k}$ ). We shall derive some consequences of Euler's formula. We concentrate on $S_{g}$, but note that similar results holds for $N_{k}$. Let $f_{i}$ be the number of facial walks of length $i$. (If an edge is traversed twice it contributes 2 to the length.) Then

$$
f_{3}+f_{4}+\cdots=f \text { and } 3 f_{3}+4 f_{4}+\cdots=2 q \text {. }
$$

Hence $2 q \geq 3 f_{3}+3 f_{4}+\cdots=3 f$ and consequently $q \leq 3 n-6+6 g$ with 
equality if $0=f_{4}=f_{5}=\cdots$ (in which case $G$ is a triangulation). The sum of degrees in $G$ equals $2 q$. So, if $G$ triangulates $S_{g}$ (or $N_{k}$ ), then $G$ has at least $3 n-6$ edges.

Also, the average degree of $G$ is at most $6+12(g-1) / n$. This implies the first part of the well-known Lemma 2.2 below.

Lemma 2.2. Let $G$ be a connected graph of genus $g$ and order $n$.

(a) If $n>12(g-1)$, then $G$ has a vertex of degree $\leq 6$.

(b) If $n>28(g-1)$ and $G$ has minimum degree at least 3 , then $G$ has girth $\leq 6$.

If (b) were false, then we have $2 q \geq 3 n$ and $2 q \geq 7 f$, which imply, by Euler's formula, $2-2 g=n-q+f \leq-q / 21 \leq-3 n / 42$, contradicting the assumption $n>28(g-1)$.

In this paper we investigate vertex-transitive graphs for which $n$ is large compared to $g$. Lemma 2.2 shows that such a graph is $d$-regular with $d \leq 6$. We shall go a little further.

Lemma 2.3. Let $G$ be a d-regular graph of order $n$ and genus $g$.

(a) If $d=6$ and $n>24(g-1)$, then, for every embedding of $G$ in $S_{g}, G$ has a vertex $v$ which is in six facial 3-cycles.

(b) If $d=5$ and $n>24(g-1)$, then $G$ has a vertex whose neighborhood graph contains either a 3-path or two disjoint paths of length 1 and 2, respectively.

(c) If $d=4, n>360(g-1)$, and $G$ has girth $>3$, then, for every embedding of $G$ in $S_{g}, G$ contains a vertex which has distance at least 3 to each face which is not bounded by a 4-cycle.

(d) If $d=3$ and $n>84(g-1)$, then, for every embedding of $G$ in $S_{g}, G$ has either a facial cycle of length $<6$ or a vertex which is in three facial 6-cycles.

Proof. If $d=6$, then

$$
\begin{aligned}
6 n & =2 q=3 f_{3}+4 f_{4}+\cdots \\
& =3 f+f_{4}+2 f_{5}+3 f_{6}+\cdots \\
& =3(2-2 g-n+q)+f_{4}+2 f_{5}+\cdots \\
& =3(2-2 g+2 n)+f_{4}+2 f_{5}+\cdots
\end{aligned}
$$

which implies

$$
6(g-1)=f_{4}+2 f_{5}+\cdots \geq \frac{1}{4}\left(4 f_{4}+5 f_{5}+\cdots\right) .
$$

The last number (in brackets) is an upper bound for the number of vertices which are on some facial walk of length $>3$. This proves $(a)$.

Assume next that $d=5$ and that $G$ is embedded in $S_{g}$. We shall prove that $G$ has a vertex $v$ such that at least three of the five 2-paths which have 
$v$ as center and are contained in facial walks are contained in 3-cycles (which, however, need not be facial). That will prove (b). So assume that at most two of the above five 2-paths are contained in 3-cycles. Then $G$ has at least $3 n$ "facial" 2-paths which are not contained in 3-cycles. Now consider any facial walk $W$ of length $r$, say, and let $m$ denote the number of the 2-paths in $W$ that are not contained in 3-cycles. In the face bounded by $W$ we add a new vertex $w$ and joint it to all vertices on the facial walk. We claim that the number $s$ of edges incident with $w$ is at least $(m+12) / 4$. This is clear if $r=3$ (and hence $m=0$ ). It is also clear if $r \geq 4$ and $W$ has no repetition of vertices since then

$$
s \geq \max (4, m) \geq(m+12) / 4 .
$$

If $W$ has repetition of vertices we complete the proof of the claim by induction on the number of vertex repetitions in $W$. (We leave the details for the reader.) The resulting graph then has $n+f$ vertices and at least $5 n / 2+(3 n+12 f) / 4$ edges. It also has at most $3(n+f)-6+6 g$ edges. Hence

$$
5 n / 2+(3 n+12 f) / 4 \leq 3(n+f)-6+6 g .
$$

This contradiction proves $(b)$.

By the same reasoning as in (a) we show under the assumption of (c) that $G$ has at most $40(g-1)$ vertices which are on some face not bounded by a 4-cycle. For each such vertex $v$ there are at most six 2-paths starting at $v$ and with an edge not in the "non-4-cycle face". Hence there are at most 360 $(g-1)$ vertices of distance $\leq 2$ to a "non-4-cycle face". (d) follows by a similar argument.

We shall make use of the fact that a vertex-transitive graph which has large order compared to the genus has no small nonplanar subgraph. In order to see this we use the result of Battle et al. [2] which implies that a graph which contains $m$ disjoint nonplanar subgraphs has genus $\geq m$. More precisely, we prove:

Lemma 2.4. If $G$ is a d-regular vertex-transitive graph, $H$ is a connected subgraph of radius $r$, and $G$ has order $>g d^{r}\left(d^{r}+1\right.$ ) (where $g$ is a natural number), then $G$ contains at least $g+1$ disjoint copies of $H$. In particular, if $H$ is nonplanar, then $g(G)>g$.

Proof. Let $H_{1}, H_{2}, \ldots, H_{m}$ be a maximal collection of pairwise disjoint subgraphs of $G$ each isomorphic to $H$. It is easy to prove, by induction on $r$, that there are at most $m|V(H)| d^{r}$ vertices in $G$ of distance $\leq r$ from $H^{\prime}=H_{1} \cup H_{2} \cup \cdots \cup H_{m}$. Similarly, $|V(H)| \leq d^{r}+1$. So, if $m \leq g$, then $G$ has a vertex $v$ of distance $>r$ from $H^{\prime}$. Since $G$ is vertex-transitive there is a subgraph $H_{m+1}$ in $G$ such that $H_{m+1}$ is isomorphic to $H$ and $v$ is a center of $H_{m+1}$. Then we can add $H_{m+1}$ to the collection $H_{1}, \ldots, H_{m}$, contradicting the maximality of $m$.

Note that the proof also applies to edge-transitive graphs if we replace $g d^{r}\left(d^{r}+1\right)$ by $g d^{r+1}\left(d^{r}+1\right)$. Using the additivity theorem of Stahl and Beineke [6], we get a nonorientable version of Lemma 2.4. 
We now consider the genus of the line graph of a cubic graph. We shall only use the trivial part, but include the nontrivial part for the sake of completeness.

Lemma 2.5. If $G$ is a connected cubic graph, then $g(L(G))=g(G)$.

Proof. It is easy to modify an embedding of $G$ in $S_{g(G)}$ to an embedding of $L(G)$ in $S_{g(G)}$. So $g(L(G)) \leq g(G)$.

To prove the converse we first make the following observation: If a connected graph $G$ has at least as many edges as vertices, then it is possible to associate with every vertex $v$ one of its incident edges $e_{v}$ such that $e_{v} \neq e_{u}$ whenever $v \neq u$. This follows easily from Hall's theorem. It can also be proved directly since it suffices to consider a connected subgraph of $G$ which has the same number of vertices and edges. Such a graph contains only one cycle and therefore the above assignment is easy to describe explicitly. Consider now the line graph of a cubic connected graph $G$. For every vertex $v$, let $e_{v}$ be one of its incident edges as above. In $L(G), e_{v}$ is a vertex which is contained in a 3-cycle $C_{v}$ corresponding to the three edges incident with $v$. Delete from $C_{v}$ the edge not incident with $e_{v}$. The resulting graph is a subdivision of $G$ in the sense that it is obtained from $G$ by replacing some edges by 2-paths. This proves that $g(G) \leq g(L(G))$.

Let $G$ be a connected $d$-regular graph $(d \geq 3)$ and $\mathscr{C}$ a collection of $m$-cycles in $G$. Assume that every edge of $G$ is contained in precisely two cycles in $\mathscr{C}$ and that, for each vertex $v$ in $G$, the edges incident with $v$ can be labelled $e_{1}, e_{2}, \ldots, e_{d}$ such that for each $i=1,2, \ldots, d$ there is a cycle in $\mathscr{C}$ containing $e_{i}$ and $e_{i+1}$ (where $e_{d+1}=e_{1}$ ). Then we can define a surface $S$ by letting the cycles of $\mathscr{C}$ be disjoint convex polygons in the Euclidean plane pasted together by the graph $G$. We shall say that $G$ and $S$ are obtained by pasting $m$-cycles together or that $G$ is a $(d, m)$-tiling of $S$. By the classification theorem, $S$ is of the form $S_{g}$ or $N_{k}$. Now $2 q=m f=d n$, where $f=|\mathscr{C}|, q=|E(G)|$, and $n=|V(G)|$. So, by Euler's formula,

$$
(d(1-2 / m)-2) n=4\left(g^{\prime}-1\right),
$$

where $g^{\prime}=g$ or $g^{\prime}=k / 2$. For fixed $g^{\prime} \neq 1$, there are only finitely many possibilities for $G$. So the case $g^{\prime}=1$ (i.e., the case where $S$ is the torus or the Klein bottle) is particularly interesting. By Lemma 2.2, we have $d \leq 6$ in this case which leaves the three possibilities $d=3$ and $m=6, d=m=4$, and $d=6$ and $m=3$. Motivated by this we now define a regular tiling of the torus or the Klein bottle as a $(d, m)$-tiling $G$, where $(d, m)$ is one of the pairs $(3,6),(4,4),(6,3)$ and the following additional conditions are imposed: If $(d, m)=(4,4), v$ is any vertex of the tiling, and $C_{1}, C_{2}, C_{3}, C_{4}$ are the four 4-cycles of the tiling containing $v$, then $C_{1} \cup C_{2} \cup C_{3} \cup C_{4}$ has nine vertices. (Note that $G$ may have girth 3 . However, it is easy to see that none of $C_{1}, C_{2}, C_{3}, C_{4}$ can have a diagonal.) If $(d, m)=(6,3)$ we require that each closed neighborhood graph is planar. (To justify this condition we note that it is natural to require that all closed neighborhood graphs be isomorphic. 
By the additivity theorems $[2,6]$ they cannot all be nonplanar if $G$ is large. Thus the omission of the planarity condition would result in only finitely many additional tilings.) Finally we require for $(d, m)=(3,6)$ that $G$ has girth 6 . (If $G$ is a $(3,6)$-tiling, then the dual $G^{\prime}$ defined below is a $(6,3)$-tiling. Now if $G^{\prime}$ is a regular tiling it turns out, as proved in Theorem 3.2 below, that $G$ has girth 6.)

Below we describe classes of graphs obtained by pasting $m$-cycles together. In the subsequent sections we then show that the list includes all regular tilings of the torus or the Klein bottle and that all the vertex-transitive graphs of large order compared to the genus can be found in this list or can be obtained from a graph in the list by simple modifications. To simplify the description, we define the dual graph $G^{\prime}$ of the above graph $G$ as the graph whose vertex set is $\mathscr{C}$ such that two vertices are adjacent iff they share an edge. Strictly speaking, duality is defined with respect to a given collection $\mathscr{C}$. However, it will always be clear from the context which collection $\mathscr{C}$ we are referring to. In most cases $\mathscr{C}$ will simply be the collection of all 6-cycles. Moreover, the graphs we are going to describe all (except perhaps some degenerate ones) have unique embeddings in the torus or the Klein bottle. (This can be proved directly or by using the results in [7].) $\mathscr{C}$ will then be the collection of facial cycles and $G^{\prime}$ is the standard geometric dual graph of $G$.

We define a quadrilateral cylinder of length $k$ and breadth $m$ as the cartesian product of a $k$-cycle and an $m$-path. If $m=1$ we call it a quadrilateral cylinder circuit of length $k$. Consider a 4-cycle $x_{1} x_{2} x_{3} x_{4} x_{1}$ in a quadrilateral cylinder circuit. Assume that the edges $x_{1} x_{2}, x_{3} x_{4}$ are in other 4-cycles as well. If we replace $x_{1} x_{4}$ and $x_{2} x_{3}$ by $x_{1} x_{3}$ and $x_{2} x_{4}$, respectively, then the resulting graph is a quadrilateral Möbius circuit of length $k$. Figure 1 shows examples of these graphs.

The cycles $x_{1} x_{2} \cdots x_{k} x_{1}$ and $y_{1} y_{2} \cdots y_{k} y_{1}$ of Figure 1 are called peripheral cycles. (The indices are expressed modulo $k$.) Note that the quadrilateral Möbius circuits have only one peripheral cycle and it has length $2 k$. If $r$ is a natural number, we denote by $Q_{k, m, r}$ the graph obtained from the quadrilateral cylinder of length $k$ and breadth $m$ by adding all edges $x_{i} y_{i+r}, i=1,2, \ldots, k$. Then $Q_{k, m, r}$ has a "natural" collection of 4-cycles (which is the set of all 4cycles when $k>4, m \geq 4$ ) which defines an embedding of $Q_{k, m, r}$ in the torus. Also, $Q_{k, m, r}$ is vertex-transitive and in some cases even 1-transitive (when $k=m+1, r=0$ ). When we speak of $Q_{k, m, r}, r$ is always a nonnegative integer. We shall also define graphs $Q_{k, m, a}, Q_{k, m, b}$, etc., where $a, b, \ldots$ are letters. All these (except $Q_{k, m, e}$ ) have natural embeddings on the Klein bottle. $Q_{k, m, a}$ is obtained from the quadrilateral cylinder above by adding the edges $x_{1} y_{2}, x_{2} y_{1}, x_{3} y_{k}, x_{4} y_{k-1}$, etc. If $k$ is even we let $Q_{k, m, b}$ be obtained by adding instead the edges $x_{1} y_{1}, x_{2} y_{k}, x_{3} y_{k-1}$, etc. $Q_{k, m, b}$ is never vertex-transitive while $Q_{k, m, a}$ is vertex-transitive iff $k=4$.

$Q_{k, m, c}$ is obtained from the above quadrilateral cylinder (with $k$ even) by adding all diagonals to the peripheral cycles. Then $Q_{k, m, c}$ is vertex-transitive iff $m=1 . Q_{k, m, c}$ may also be thought of as obtained from a quadrilateral 


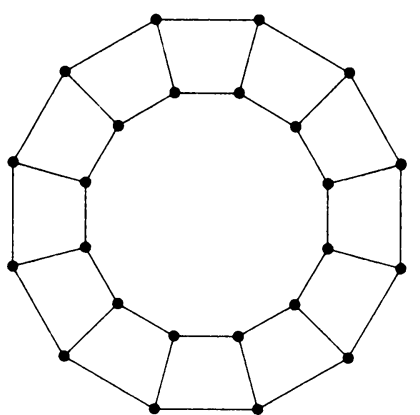

(a)

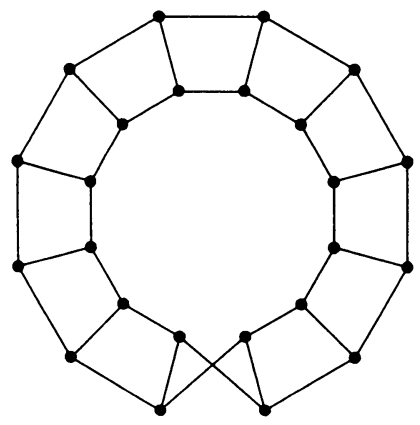

(b)

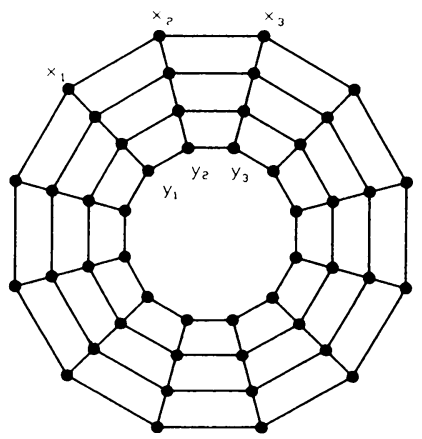

(c)

FIGURE 1. A quadrilateral cylinder (respectively Möbius) circuit of length 12 and a quadrilateral cylinder of length 12 and breadth 3 .
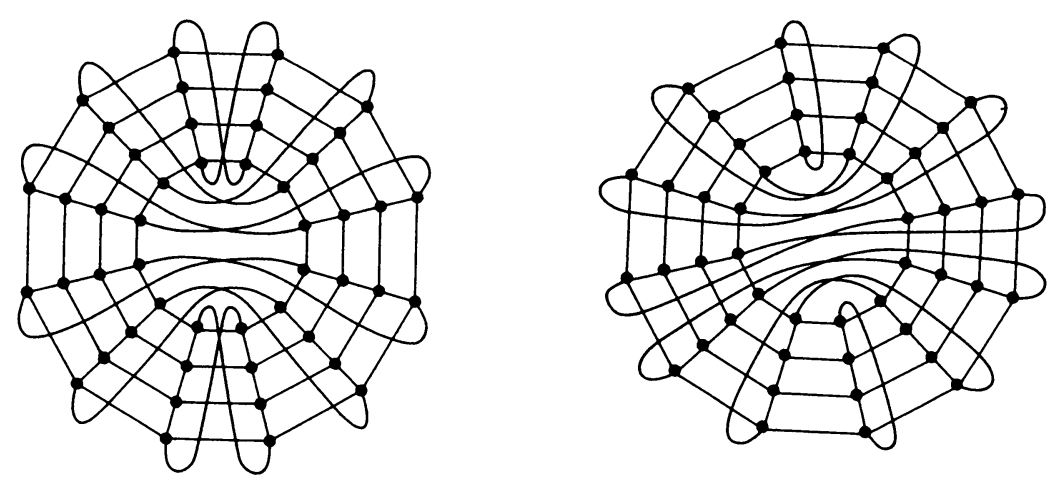

FIGURE 2. $Q_{12,3, a}$ and $Q_{12,3, b}$

cylinder of length $k$ and two quadrilateral Möbius circuits of length $k / 2$ by identifying each peripheral cycle in the cylinder with a peripheral cycle in one of the Möbius circuits. We define a quadrilateral Möbius double circuit of length 


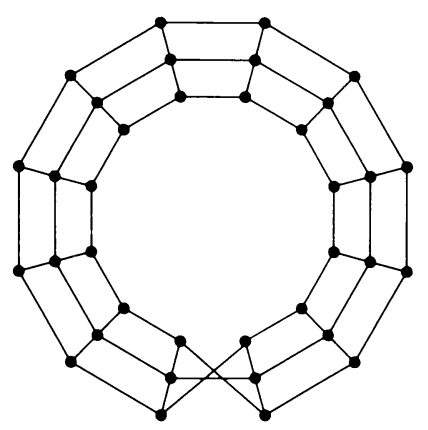

Figure 3. A quadrilateral Möbius double circuit of length 12

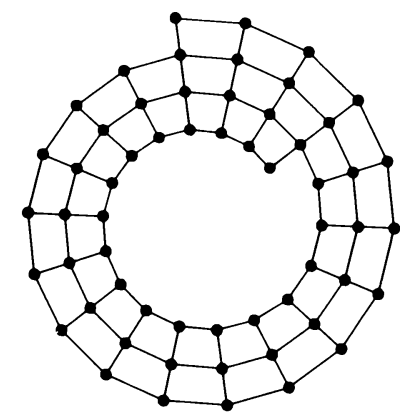

FIGURE 4. Part of $Q_{58,18, e}$

$k$ as a graph obtained from a quadrilateral cylinder of length $k$ and breadth 2 by switching two edges which are on distinct peripheral cycles and which are in a common 6-cycle as indicated in Figure 3.

Now define $Q_{k, m, f}$ ( $k$ even, $k \geq 6$ ) as the graph obtained from the quadrilateral cylinder of length $k$ and breadth $m$ by identifying each peripheral cycle with the peripheral cycle in a Möbius double cycle. We shall also allow $m$ to be zero here, i.e., $Q_{k, 0, f}$ is obtained by pasting two Möbius double cycles together along the peripheral cycle. None of the graphs $Q_{k, m, f}$ are vertex-transitive. If $k$ is even, then $Q_{k, m, g}$ is the graph obtained from the cylinder by pasting a quadrilateral Möbius circuit (respectively Möbius double circuit) on one (respectively the other) peripheral cycle. The degenerate $Q_{k, 0, g}$ is isomorphic to $Q_{3, k / 2-1, a}$. None of $Q_{k, m, g}$ are vertex-transitive.

We define the graph $Q_{k, m, e}$ as the graph obtained from a cycle $x_{1} x_{2} \cdots x_{k} x_{1}$ by adding all edges $x_{i} x_{i+m}$. Clearly, $Q_{k, m, e}$ is vertex-transitive and has a natural embedding in the torus. Part of $Q_{58,18, e}$ is shown in Figure 4.

Finally we define $Q_{k, m, h}$ as follows: First we take a graph which is the Cartesian product of two $(m-1)$-paths. Let $x_{1} x_{2} \cdots x_{m}, y_{1} y_{2} \cdots y_{m}, u_{1} u_{2} \cdots u_{m}$, and $v_{1} v_{2} \cdots v_{m}$ be paths in this product such that $x_{m}=y_{1}, x_{1}=u_{m}, v_{1}=u_{1}$, $y_{m}=v_{m}$, and $v_{1}, x_{1}, y_{1}, y_{m}$ have degree 2 and all other vertices $x_{i}, y_{i}, v_{i}, u_{i}$ 


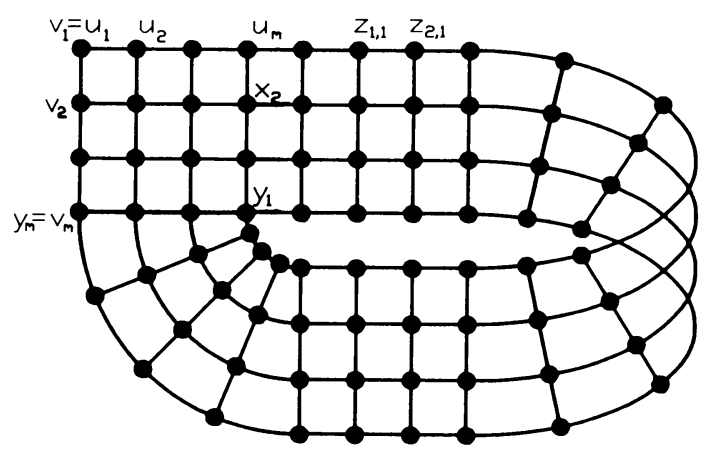

Figure 5. Part of $Q_{15,4, h}$
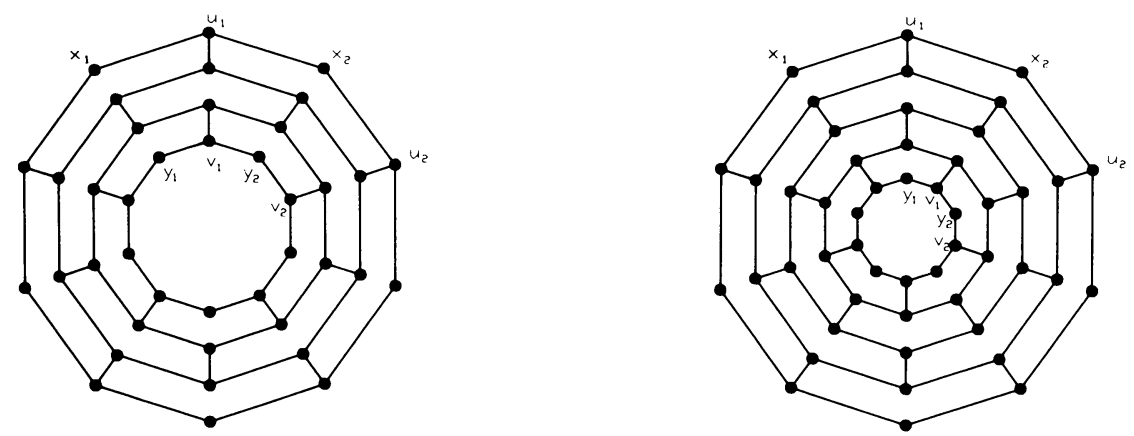

FIGURE 6. Hexagonal cylinders of length 5 and breadth 3 and 4 , respectively

have degree 3 . Then add paths

$$
x_{1} z_{1,1} z_{2,1} \cdots z_{k, 1} y_{1}, x_{2} z_{1,2} z_{2,2} \cdots z_{k, 2} y_{2}, \ldots, x_{m} z_{1, m} z_{2, m} \cdots z_{k, m} y_{m}
$$

such that all the new vertices $z_{i, j}$ are distinct. Add the paths $z_{i, 1} z_{i, 2} \cdots z_{i, m}$, where $i=1,2, \ldots, k$. This graph is indicated in Figure 5 .

Then add all edges $u_{1} z_{1, m}, u_{2} z_{2, m}, \ldots, z_{k-1,1} v_{2}, z_{k, 1} u_{1}$. None of the graphs $Q_{k, m, h}$ are vertex-transitive.

We now define graphs pasted together with 6-cycles. A hexagonal cylinder of length $k$ and breadth $m$ is defined analogous to the quadrilateral cylinder. Examples are shown in Figure 6.

A hexagonal cylinder circuit is a hexagonal cylinder of breadth 1 . A hexagonal Möbius circuit (or double circuit) is defined as indicated in Figure 7.

The peripheral cycles are those where every second vertex has degree 2.

Now $H_{k, m, r}$ is obtained from a hexagonal cylinder of length $k$ and breadth $m$ by adding all edges $x_{i} y_{i+r}$ (see Figure 6). It is easy to see that $H_{k, m, r}$ is vertex-transitive and (for some values of $k, m, r$ ) even 1 -transitive. $H_{k, m, a}$ is obtained by adding the edges $y_{1} x_{2}, y_{2} x_{1}, y_{3} x_{k}$, etc. For $k$ even and $m$ odd, 

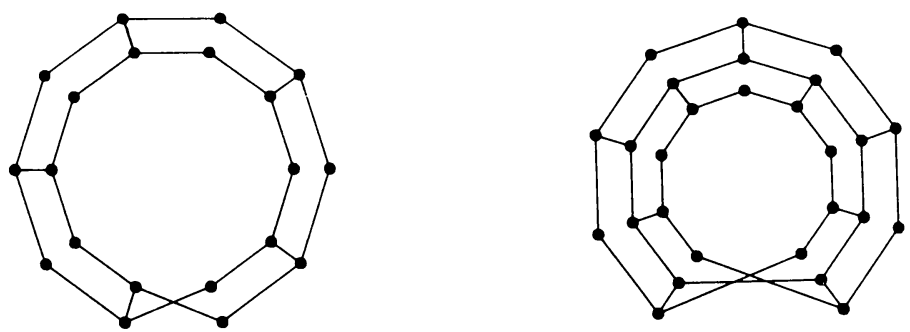

FIGURE 7. A hexagonal Möbius circuit of length 5, and a hexagonal Möbius double circuit of length 9

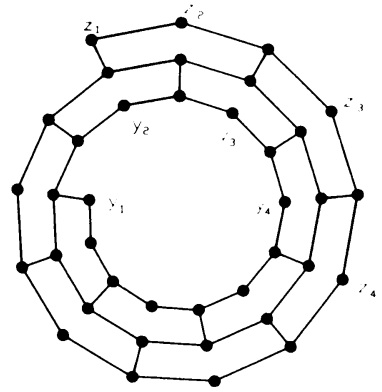

FIGURE 8

$H_{k, m, b}$ is obtained by adding the edges $y_{1} x_{1}, y_{2} x_{k}$, etc. $H_{k, m, b}$ is not vertextransitive (except for $H_{2, m, b}=H_{2, m, 0}$ ), and $H_{k, m, a}$ is not vertex-transitive (except for $H_{2, m, a}=H_{2, m, 1}$ and for $k=4$ ).

For $k$ even, $H_{k, m, c}$ is obtained from a hexagonal cylinder of length $k$ and breadth $m$ by adding all diagonals of the form $x_{i} x_{i+k / 2}, y_{i} y_{i+k / 2}$ to the peripheral cycles. If $k$ is odd, $H_{k, m, f}$ is obtained by adding a star cycle with respect to each peripheral cycle. That is, we add new vertices $w_{1}, w_{2}, \ldots, w_{k}$ and the edges $w_{i} x_{i}$ for $i=1,2, \ldots, k$ and the cycle $w_{i} w_{i+(k+1) / 2} w_{i+1} w_{i+(k+3) / 2} \cdots$. A similar cycle is added to the other peripheral cycle. The graphs $H_{k, m, c}, H_{k, m, f}$ are not vertex-transitive (when $m \geq 1$ ). For $k$ odd, $H_{k, d}$ (which is also the degenerate $\left.H_{k, 0, f}\right)$ is obtained by pasting two Möbius double circuits together along their peripheral cycle such that the resulting graph is cubic. Note that $H_{k, d}$ is not vertex-transitive but its dual graph is. Finally, the graph $H_{k, m, e}$ which is vertex-transitive and toroidal is defined as follows: We first form a "hexagonal spiral" as indicated in Figure 8. This is analogous to the quadrilateral "spiral" in Figure 4. Then we add the edges $z_{1} y_{1}, z_{2} y_{2}, z_{3} y_{3}, \ldots$ The graphs $H_{k, m, e}$ are all vertex-transitive.

We have now defined families of graphs obtained by pasting 4-cycles or 6cycles together. Some of these are vertex-transitive. Some are even 1-transitive. Using the 1-transitive ones we can construct new vertex-transitive graphs on the torus or the Klein bottle using one of the operations (a)-(e) in Figure 9.

In Figure 9(f), (g) we only add edges. These are indicated by dotted lines. Note that the operations in $(\mathrm{f}),(\mathrm{g})$ can only be performed in special cases of the 


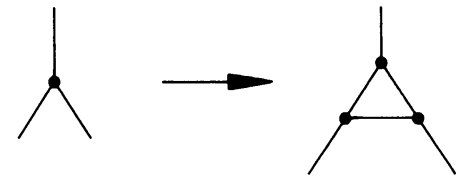

(a)

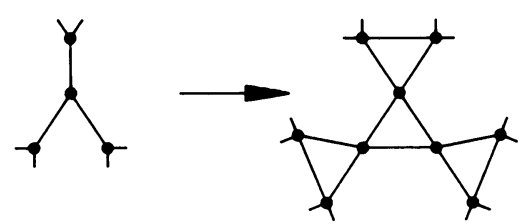

(c)

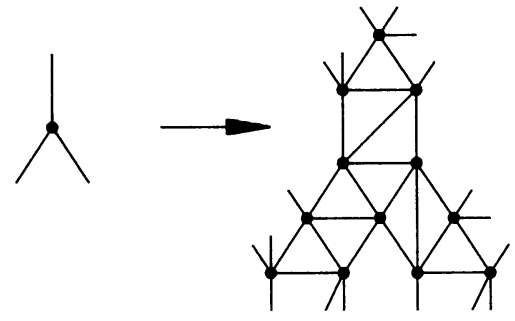

(e)

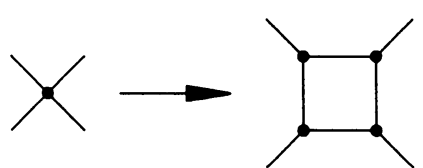

(b)

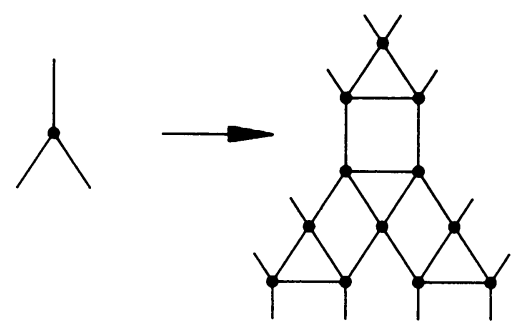

(d)

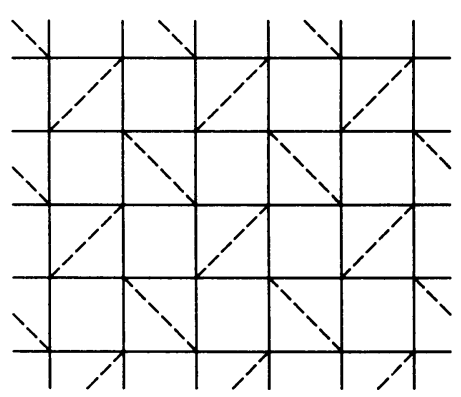

(f)

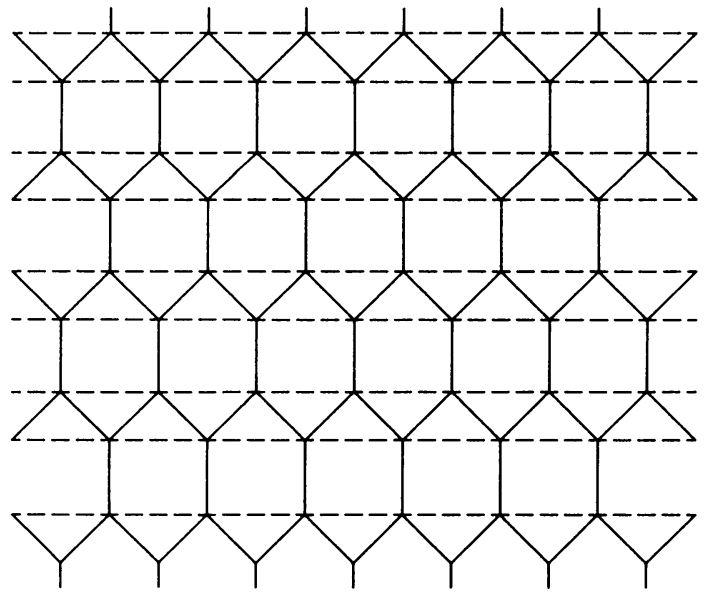

(g)

Figure 9 
graphs previously defined. Whenever the operation can be performed and the initial graph is vertex-transitive, then also the resulting graph is vertex-transitive.

\section{HeXagonal tilings and triangulations OF THE TORUS AND THE KLEIN BOTTLE}

In this section we characterize those cubic graphs of girth 6 which are obtained by pasting 6-cycles together. Although the result and its proof are purely combinatorial it may be convenient to think of the 6-cycles as polygons pasted together such that they form a torus or a Klein bottle as pointed out in $\S 2$.

Theorem 3.1. Let $G$ be a connected cubic graph of girth 6 and $\mathscr{C}$ a collection of 6-cycles in $G$ such that every 2-path in $G$ is contained in precisely one cycle of $\mathscr{C}$. Then for some natural numbers $k, m$, and $r, G$ is isomorphic to one of

$$
H_{k, m, r}, H_{k, m, a}, H_{k, m, b}, H_{k, m, c}, H_{k, d}, H_{k, m, e}, H_{k, m, f} .
$$

Proof. The assumption of Theorem 3.1 implies that every edge of $G$ is in precisely two 6-cycles in $\mathscr{C}$ having precisely $e$ (and its ends) in common.

Assume first that $G$ has a hexagonal cylinder circuit of length $k \quad(k \geq 3)$ such that each nonperipheral 6-cycle is in $\mathscr{C}$. Extend this hexagonal circuit to a maximal hexagonal cylinder $H$ of breadth $m$, say, such that each nonperipheral 6-cycle of $H$ is in $\mathscr{C}$. Let $C_{1}, C_{2}$ be the two peripheral cycles of $H$. If some vertex $x$ (of $H$-degree 2) in $C_{1}$ is joined to some vertex $y$ of $C_{2}$ by an edge $e$, then the two 6-cycles in $\mathscr{C}$ containing $e$ show that each vertex of distance 2 from $e$ on $C_{1}$ is joined to a vertex of distance 2 from $y$ on $C_{2}$. Repeating this argument we conclude that $G$ is obtained from $H$ by adding edges between $C_{1}$ and $C_{2}$. Now it is easy to see that $G$ is of the form $H_{k, m, a}$ or $H_{k, m, b}$ or $H_{k, m, r}$. So assume that no vertex of $C_{1}$ (of $H$-degree 2) is joined to $C_{2}$.

If some vertex $x$ in $H$-degree 2 in $C_{1}$ is joined to another vertex $y$ of $H$-degree 2 in $C_{1}$ by an edge $e$, then, as above, we consider the two 6-cycles in $\mathscr{E}$ containing $e$ and conclude that every vertex of $H$-degree 2 in $C_{1}$ is joined to a vertex of $H$-degree 2 in $C_{1}$. Since $G$ is cubic and has girth 6 , $k$ must be even and $x$ and $y$ must be diametrically opposite on $C_{1}$. If no vertex of $H$-degree 2 in $C_{1}$ is joined to a vertex of $H$-degree 2 in $C_{1}$, then each vertex $x$ of $H$-degree 2 in $C_{1}$ is joined to a vertex $x^{\prime}$ in $V(G) \backslash V(H)$. The 2-path condition in Theorem 3.1 implies that $x^{\prime} \neq y^{\prime}$ for $x \neq y$. Let $C_{1}: x_{1} u_{1} x_{2} u_{2} \cdots x_{k} u_{k} x_{1}$ such that $x_{1}, x_{2}, \ldots, x_{k}$ have $H$-degree 2 and the indices are expressed modulo $k$. We have previously observed that the vertices $x_{1}^{\prime}, x_{2}^{\prime}, \ldots, x_{k}^{\prime}$ are distinct and outside $H$. The 6-cycles in $\mathscr{C}$ containing $x_{i} x_{i}^{\prime}$ are of the form $x_{i}^{\prime} x_{i} u_{i} x_{i+1} x_{i+1}^{\prime} z_{i} x_{i}^{\prime}$ and $x_{i}^{\prime} x_{i} u_{i-1} x_{i-1} x_{i-1}^{\prime} z_{i-1} x_{i}^{\prime}$. Since $G$ is cubic, none of the vertices $z_{i}$ are in $H$. The maximality of $m$ implies that at least one of them is in $\left\{x_{1}^{\prime}, x_{2}^{\prime}, \ldots, x_{k}^{\prime}\right\}$, say $z_{1}=x_{i}^{\prime}$. The 6-cycle in $\mathscr{C}$ which contains $x_{2}^{\prime} x_{i}^{\prime}$ (and avoids $x_{1} x_{1}^{\prime}$ ) must contain $x_{i}^{\prime} x_{i}$ and hence it must be one of $x_{2}^{\prime} x_{i}^{\prime} x_{i} u_{i} x_{i+1} x_{i+1}^{\prime} x_{2}^{\prime}$ or $x_{2}^{\prime} x_{i}^{\prime} x_{i} u_{i-1} x_{i-1} x_{i-1}^{\prime} x_{2}^{\prime}$. It must be the former since otherwise the 6-cycle in $\mathscr{C}$ containing $x_{2}^{\prime} x_{i-1}^{\prime}$ and avoiding $x_{2}^{\prime} x_{i}^{\prime}$ 
implies the presence of the edge $x_{3}^{\prime} x_{i-1}^{\prime}$ and (by repeating the argument) the edges $x_{3}^{\prime} x_{i-2}^{\prime}, x_{i-2}^{\prime} x_{4}^{\prime}$ which finally leads to a contradiction. So $G$ contains the edge $x_{2}^{\prime} x_{i+1}^{\prime}$ and (by repeating the argument) the edges $x_{i+1}^{\prime} x_{3}^{\prime}, x_{3}^{\prime} x_{i+2}^{\prime}$, etc. Hence $k$ must be odd and $i=(k+1) / 2$. The cycle $x_{1}^{\prime} x_{(k+1) / 2}^{\prime} x_{2}^{\prime} x_{(k+3) / 2}^{\prime} \cdots$ is a star cycle with respect to $C_{1}$. Summarizing, $G$ is obtained from $H$ by joining diametrically opposite vertices of $H$-degree 2 in $C_{1}$ or $C_{2}$ or by adding two star cycles to the vertices of $H$-degree 2 in $C_{1}$ and $C_{2}$, respectively. In any case $G$ is of the form $H_{k, m, c}$ or $H_{k, m, f}$. So, we assume that $G$ has no hexagonal cylinder circuit.

Assume next that $G$ has a hexagonal Möbius double circuit $H$ of length $2 k-1 \quad(k \geq 4)$ whose 6 -cycles are in $\mathscr{C}$. Let $C$ be the peripheral cycle of $H$. Consider those 6-cycles in $\mathscr{C}$ which have edges in $H$ and also edges outside of $H$. By the above assumption, these do not form a hexagonal cylinder circuit. Also, each of them has only a 2-path in common with $H$ because no 2-path is in two cycles in $\mathscr{C}$. By an argument similar to one of a previous case we conclude that $G$ is obtained from $H$ by adding a star cycle with respect to $C$, that is, $G$ is isomorphic to $H_{k, d}$. So, we can assume that $G$ has neither a hexagonal cylinder circuit nor a hexagonal Möbius double circuit.

Assume now that $G$ has a hexagonal Möbius circuit $H$ of length $k$ and with peripheral cycle $C$. If some two vertices in $C$ of $H$-degree 2 are adjacent, then, by the same reasoning as in previous cases, every vertex $x$ of $H$-degree 2 in $H$ is joined to its diametrically opposite vertex $y$ in $C$. However, $x$ and $y$ are in the same 6-cycle, which contradicts the assumption that $G$ has girth 6 . As in previous cases we conclude that $G$ is obtained from $H$ by adding a star-cycle to the vertices of $H$-degree 2. But, this is impossible since $H$ has an even number of vertices of degree 2. So we can assume that $G$ has no hexagonal Möbius circuit either.

We now define a sequence of 6-cycles $C_{i}: x_{1, i} x_{2, i} x_{3, i} x_{4, i} x_{5, i} x_{6, i} x_{1, i}$ in $\mathscr{C}$ as follows. Let $C_{1}$ be any cycle in $\mathscr{C}$. Having defined $C_{i}$ we let $C_{i+1}$ be the cycle in $\mathscr{C} \backslash\left\{C_{i}\right\}$ such that $x_{1, i+1}=x_{5, i}$ and $x_{2, i+1}=x_{4, i}$. Let $k$ be the smallest number such that $C_{k}$ has more than a 1-path in common with $C_{1} \cup C_{2} \cup \cdots \cup C_{k-1}$. Choose $C_{1}, C_{2}, \ldots$ such that $k$ is minimum. Then $C_{k}$ has nothing in common with $C_{2} \cup C_{3} \cup \cdots \cup C_{k-2}$, and $C_{k} \cap C_{1}$ is a 1-path in $x_{6,1} x_{1,1} x_{2,1} x_{3,1}$. Since $G$ contains no hexagonal cylinder circuit or Möbius circuit, we can assume that $C_{1} \cap C_{k}$ is not both the path $x_{1,1} x_{2,1}$ and the path $x_{4, k} x_{5, k}$. We can assume that $C_{1} \cap C_{k}$ is the path $x_{1,1} x_{6,1}$. (For if $C_{1} \cap C_{k}=x_{1,1} x_{2,1}$ then we consider the sequence $C_{k}, C_{1}, C_{2}, \ldots$ instead of $C_{1}, C_{2}, \ldots$ ) If $x_{3, k}=x_{1,1}$ and $x_{4, k}=x_{6,1}$, then we consider the sequence $C_{1}, C_{2}, \ldots, C_{k}, C_{k+1}, \ldots$. For each $i \geq k, C_{i+1}$ has (at least) a 3-path in common with $C_{1} \cup \cdots \cup C_{i}$. Let $m$ be the smallest number such that $C_{m+1}$ has more than a 3-path in common with $C_{1} \cup \cdots \cup C_{m}$. Then $C_{m+1}$ contains the edge $x_{2,1} x_{3,1}$ and one of the edges $x_{6, m-k+2} x_{2,1}, x_{6, m-k+2} x_{3,1}$. It must be the latter since the former cannot be in two cycles in $\mathscr{C}$. The other 6-cycle 
in $\mathscr{E}$ containing $x_{6, m-k+2} x_{3,1}$ also contains $x_{6, m-k+3} x_{3,2}$. Repeating this, we conclude that $G$ contains all edges $x_{6, m-k+4} x_{3,3}, x_{6, m-k+5} x_{3,4}$, etc. It follows that $G$ is of the form $H_{k, m, e}$.

If $x_{6, k}=x_{1,1}$ and $x_{5, k}=x_{6,1}$, then we consider $C_{1} \cup C_{2} \cup \cdots \cup C_{2 k-1}$. Since this is not a hexagonal Möbius double circuit, there is a smallest $m<2 k-2$ such that $C_{m+1}$ has more than a 3-path in common with $C_{1} \cup \cdots \cup C_{m}$. By reasoning as in the previous case we obtain a contradiction to the assumption that $G$ is cubic and satisfies the 2-path condition.

We are thus left with the cases where $x_{1,1} x_{6,1}$ is one of the paths $x_{4, k} x_{5, k}$, $x_{5, k} x_{4, k}, x_{5, k} x_{6, k}, x_{4, k} x_{3, k}$. Let $C_{1,1}$ be the 6-cycle in $\mathscr{C}$ containing $x_{6,1} x_{5,1} x_{6,2}$, and let $C_{2,1}$ be the 6-cycle in $\mathscr{C}$ containing $x_{6,2} x_{5,2} x_{6,3}$. Continuing like this we define a sequence $C_{1,1}, C_{2,1}, \ldots$ which we consider instead of $C_{1}, C_{2}, \ldots$ By the minimality of $k$, each $C_{i, 1}(2 \leq i<k)$ has only a 1 path in common with $C_{1,1} \cup \cdots \cup C_{i-1,1}$. Hence $C_{1} \cup \cdots \cup C_{k}$ cannot be planar. (Intuitively, it is a Möbius strip.) So, $x_{6,1} x_{1,1}$ is one of the paths $x_{4, k} x_{5, k}$ or $x_{3, k} x_{4, k}$. We shall show that this leads to a contradiction. We first assume that $x_{1,1}=x_{5, k}$ and $x_{6,1}=x_{4, k}$. We consider again $C_{1,1}, C_{2,1}, \ldots, C_{k, 1}$ and conclude that $C_{k, 1}$ is the 6-cycle in $\mathscr{C} \backslash\left\{C_{1}\right\}$ containing $x_{1,1} x_{2,1}$. Then we consider the sequence $C_{1,2}, C_{2,2}, \ldots, C_{k, 2}$, where $C_{1,2}$ is the cycle in $\mathscr{C}$ containing the 2-path $x_{3, k-1} x_{2, k} x_{3, k}$ such that $C_{i, 2}$ has an edge in common with both $C_{i, 1}$ and $C_{i-1,2}$ for $i=2, \ldots, k$. In a similar way we define the sequence $C_{1,3}, C_{2,3}, \ldots, C_{k, 3}$ of cycles in $\mathscr{C}$, etc. Let $C_{i, m+1}$ be the first cycle which has more than a 3-path in common with the previously defined 6-cycles. We must have $i=1$, since otherwise we get a contradiction as in previous reasoning. Then $C_{1, m+1}$ has an edge in common with $C_{k, m}$ and hence the sequence $C_{k, m}, C_{k-1, m-1}, \ldots, C_{1, m+1}$ forms a hexagonal cylinder (or Möbius) circuit. This is a contradiction.

We consider finally the case where $x_{6,1}=x_{3, k}$ and $x_{1,1}=x_{4, k}$. For each $m, m=1,2, \ldots,\lfloor(k+1) / 2\rfloor$, we consider the sequence $C_{1, m}, C_{2, m}, \ldots$ of 6-cycles in $\mathscr{C}$, where $C_{1, m}=C_{m}, C_{2, m}$ is the 6-cycle in $\mathscr{C} \backslash\left\{C_{m}\right\}$ containing $x_{6, m} x_{1, m}, C_{3, m}$ is the 6-cycle in $\mathscr{C} \backslash\left\{C_{2, m}\right\}$ containing the edge of $C_{2, m}$ opposite to $x_{6, m} x_{1, m}$, etc. It is easy to prove, by induction on $m$, that $C_{m+1, m}=C_{k+1-m}$. So, for $m=\lfloor(k+1) / 2\rfloor$ we get a contradiction to the minimality of $k$. This completes the proof.

Theorem 3.1 implies the following:

Theorem 3.2. Let $G^{\prime}$ be a connected 6-regular graph and $\mathscr{C}^{\prime}$ a collection of 3cycles in $G^{\prime}$ such that, for every vertex $v$ of $G^{\prime}$, there are precisely six cycles in $\mathscr{C}^{\prime}$ that contain $v$ and their union is a 6-wheel $W_{v}$ with $v$ as center. Suppose further that $G^{\prime}$ has no nonplanar subgraph of radius 1 . Then $G^{\prime}$ is a dual graph of one of the graphs described in Theorem 3.1 .

Proof. We form a graph $G$ as follows: $V(G)=\mathscr{C}^{\prime}$, and two vertices of $G$ are adjacent if and only if the corresponding cycles in $\mathscr{C}^{\prime}$ have an edge in 
common. Let $\mathscr{C}$ denote the 6-cycles in $G$ corresponding to the 6-wheels in $G^{\prime}$ of the form $W_{v}$. Then clearly $G$ is connected, cubic, and every 2-path in $G$ is contained in a 6 -cycle in $\mathscr{C}$. In order to obtain Theorem 3.2 from Theorem 3.1 it only suffices to show that a 2-path in $G$ is contained in only one 6-cycle in $\mathscr{C}$ and in no cycle of length $<6$. Let $t_{1} t_{2} t_{3}$ be a 2-path in $G$. Then $G^{\prime}$ has a vertex $v$ and a cycle $v_{1} v_{2} v_{3} v_{4} v_{5} v_{6}$ (the indices being expressed modulo 6) such that the 3-cycles in $\mathscr{C}^{\prime}$ incident with $v$ are $t_{i}=v v_{i} v_{i+1} v$ $(i=1,2, \ldots, 6)$. Suppose (reductio ad absurdum) that $t_{1} t_{2} t_{3}$ is contained in an $m$-cycle $C(m \leq 6)$ other than $t_{1} t_{2} \cdots t_{6} t_{1}$ and that $C$ is in $\mathscr{C}$ if $m=6$. Let $C: t_{1} t_{2} t_{3} t_{4}^{\prime} \cdots t_{m}^{\prime} t_{1}$. Since $C \neq t_{1} t_{2} t_{3} t_{4} t_{5} t_{6} t_{1}$, we can assume that $t_{4}^{\prime} \neq t_{4}$, i.e., $t_{4}^{\prime}$ is of the form $v_{3} v_{4} u v_{3}$, where $u \neq v$. Since $t_{1} \cap t_{2} \cap t_{3} \cap t_{4}^{\prime}=\varnothing$, $C$ is not in $\mathscr{C}$. Hence $m \leq 5$. Since $t_{1}$ and $t_{4}^{\prime}$ have no edge in common, $m=5$. Moreover, $t_{5}^{\prime}$ is of the form $v_{1} v_{2} z v_{1}$, where $z \neq v$. Since $t_{4}^{\prime} \cap t_{5}^{\prime}$ is a 1-path, we conclude that $u \in\left\{v_{1}, v_{2}\right\}$ and $z^{\prime} \in\left\{v_{3}, v_{4}\right\}$. Since $\bar{N}(v)$ induces a planar subgraph, we cannot have $u=v_{1}$ and $z=v_{4}$. Hence one of $t_{4}^{\prime}, t_{5}^{\prime}$ (say the former) contains three consecutive vertices (namely $v_{2}, v_{3}, v_{4}$ ) of $W_{v}-v$. But then the union of the 3-cycles in $\mathscr{C}^{\prime}$ containing $v_{3}$ is not a 6-wheel. This contradiction completes the proof.

\section{Quadrilateral tilings of the torus AND the Klein bottle}

In this section we use the methods of $\S 3$ to characterize the regular tilings of the torus and the Klein bottle into quadrilaterals.

Theorem 4.1. Let $G$ be a connected 4-regular graph and $\mathscr{C}$ a collection of 4cycles in $G$ such that, for each vertex $v$ in $G$, there are precisely four cycles in $\mathscr{C}$ containing $v$ and the union of these 4-cycles form a subgraph of order 9 which is obtained from the 4-wheel with $v$ as center by subdividing each edge not incident with $v$. Then $G$ is one of the graphs $Q_{k, m, r}, Q_{k, m, a}, Q_{k, m, b}$, $Q_{k, m, c}, Q_{k, m, e}, Q_{k, m, f}, Q_{k, m, g}$, or $Q_{k, m, h}$.

Proof. As the proof is very similar to the proof of Theorem 3.1 (and in fact easier since 4-cycles are easier to deal with than 6-cycles) we shall only sketch the proof and leave the details for the reader.

We first assume that $G$ has a quadrilateral cylinder circuit. We extend it to a maximal quadrilateral cylinder. Then we conclude (in a way similar to Theorem 3.1) that $G$ is of the form $Q_{k, m, r}, Q_{k, m, a}, Q_{k, m, b}, Q_{k, m, c}, Q_{k, m, f}$ or $Q_{k, m, g}$.

Next we assume that $G$ has a quadrilateral Möbius double circuit and conclude that $G$ either has a quadrilateral circuit (which is the previous case) or $G$ is of the form $Q_{k, m, g}$ or $Q_{k, m, f}$ with $m=0$. If $G$ has a quadrilateral Möbius circuit, then either it has a quadrilateral cylinder circuit or $G$ is of the form $Q_{3, m, a}$.

So, we can assume that $G$ does not contain a quadrilateral cylinder circuit or a quadrilateral Möbius circuit (or double circuit). Then we consider a sequence 
$C_{1}, C_{2}, \ldots, C_{k}$ such that each $C_{i}$ is a cycle in $\mathscr{C}, C_{1} \cup C_{2} \cup \cdots \cup C_{k-1}$ and $C_{2} \cup$ $\cdots \cup C_{k}$ are the Cartesian products of a 1-path and a $(k-2)$-path, and $C_{1} \cup C_{k} \neq$ $\varnothing$. (We choose $C_{1}, C_{2}, \ldots, C_{k}$ such that $k$ is minimum.) Since $G$ contains no quadrilateral cylinder (or Möbius) circuit, $C_{1} \cap C_{k}$ is not a 1-path disjoint from $C_{2} \cup C_{k-1}$. If $C_{1} \cap C_{k}$ is a vertex, then we define $C_{k+1}, C_{k+2}, \ldots$ in the obvious way. Since $G$ contains no Möbius double circuit, either $C_{1}, C_{2}, \ldots$ form a pattern as in Figure 4 (in which case $G$ is of the form $Q_{m, k, e}$ ), or else $C_{1} \cap C_{k}$ is a 1-path intersecting precisely one of $C_{2}, C_{k-1}$. In the latter case we conclude as in the proof of Theorem 3.1 that $C_{1} \cup \cdots \cup C_{k}$ is not planar. (Intuitively, it is a Möbius strip.) As in the proof of Theorem 3.1, we paste successively 4-cycles in $\mathscr{C}$ on $C_{1} \cup \cdots \cup C_{k}$ as long as possible. In Theorem 3.1 the corresponding argument resulted in a contradiction. In Theorem 4.1 it shows that $G$ is of the form $Q_{k, m, h}$.

\section{EDGE-TRANSITIVE AND 1-TRANSITIVE GRAPHS OF LARGE ORDER COMPARED TO THE GENUS}

We shall apply the results of $\S \S 3$ and 4 to investigate vertex-transitive graphs. We begin with cubic edge-transitive graphs.

Proposition 5.1. Let $G$ be a connected cubic edge-transitive graph of girth $\leq 6$ such that every subgraph of $G$ of radius $\leq 4$ is planar. Then $G$ is the graph of the tetrahedron $K_{4}$, or the 3-cube $K_{2} \times K_{2} \times K_{2}$, or the dodecahedron, or one of the graphs $H_{k, m, r}$.

Proof. If $G$ has girth 3 , then $G$ is isomorphic to $K_{4}$. If $G$ has girth 4 , then $G$ is isomorphic to the graph of the 3-cube.

Assume that $G$ has girth 5 . Then $G$ is nonbipartite and hence 1-transitive by Lemma 2.1(c). Then every 2-path $x_{1} x_{2} x_{3}$ of $G$ is contained in a 5-cycle. To see this we let $x_{4}$ be the third neighbor of $x_{2}$ and we consider an automorphism taking $x_{2} x_{4}$ to an edge which is incident with (but not contained in) a given 5-cycle.

Assume that some (and hence every) 2-path $x_{1} x_{2} x_{3}$ is contained in two 5cycles $C_{1}: x_{1} x_{2} x_{3} x_{4} x_{5} x_{1}$ and $C_{2}: x_{1} x_{2} x_{3} y_{4} y_{5} x_{1}$. Then $\left\{x_{4}, x_{5}\right\} \cap\left\{y_{4}, y_{5}\right\}=$ $\varnothing$ because $G$ has girth 5 . Let $y_{2}$ be the neighbor of $x_{2}$ such that $y_{2} \notin$ $\left\{x_{1}, x_{3}\right\}$. The 2-path $x_{1} x_{2} y_{2}$ is contained in two 5-cycles $C_{1}^{\prime}, C_{2}^{\prime}$ having only $x_{1} x_{2} y_{2}$ in common. Then $C_{1} \cup C_{2} \cup C_{1}^{\prime} \cup C_{2}^{\prime}$ contains a subdivision of $K_{3,3}$ of radius 2 , a contradiction.

We can now assume that every 2-path of $G$ is contained in precisely one 5cycle. If $v$ is any vertex of $G$, then $G$ has precisely three 5-cycles $C_{1}, C_{2}, C_{3}$ containing $v$. These 5 -cycles have precisely a 2 -path in common pair by pair. Using the fact that every 2-path is in precisely one 5-cycle we conclude that there are six 5-cycles $C_{4}, \ldots, C_{9}$ which are not in $C_{1} \cup C_{2} \cup C_{3}$ and which have an edge in common with $C_{1} \cup C_{2} \cup C_{3}$. Now $C_{1} \cup C_{2} \cup \cdots \cup C_{9}$ has only three vertices of degree 2 and hence $G-\left(C_{1} \cup \cdots \cup C_{9}\right)$ must be a single vertex. It follows that $G$ is the graph of the dodecahedron. 
Assume finally that $G$ has girth 6 . Then some (and hence each) 2-path of $G$ is contained in a 6-cycle. (This is clear if $G$ is 1-transitive. If $G$ is not 1-transitive, then $G$ is bipartite and each vertex set in the bipartition is an orbit under the automorphism group of $G$. Hence every 6-cycle intersects both orbits. Using this it is easy to find a 6-cycle through any vertex and hence through any 2-path.) Suppose first that some 3-path $P_{1}$ is contained in two 6-cycles $P_{1} \cup P_{2}$ and $P_{1} \cup P_{3}$, say. Since $G$ has girth $6, P_{2}$ and $P_{3}$ have only the ends $x$ and $y$ in common. Let $P_{i}: x x_{i} y_{i} y$ for $i=1,2,3$ and put $H=P_{1} \cup P_{2} \cup P_{3}$. We say that two vertices of $G$ are mates if they are joined by three internally disjoint paths of length 3 . If $y^{\prime}$ is a mate of $x$ other than $y$ and $P_{1}^{\prime}, P_{2}^{\prime}, P_{3}^{\prime}$ are internally disjoint $x-y^{\prime}$ paths, then $H \cup P_{1}^{\prime} \cup P_{2}^{\prime} \cup P_{3}^{\prime}$ is a nonplanar graph of radius $\leq 3$, a contradiction. So $x$ (and hence every other vertex as well) has precisely one mate. Since $H$ has diameter 3 and $G$ has girth 6, every path in $G$ which joins two vertices in $H$ is either contained in $H$ or has length at least 3. Since some 2-path is contained in two 6-cycles (and if $G$ is bipartite the midpoint of the 2-path can be chosen to be in any partite class), it follows that every 2-path is in two 6-cycles. For each pair $i, j$ in $\{1,2,3\}$ let $C_{i, j}$ be a 6-cycle not in $H$ containing the path $x_{i} x x_{j}$. By the preceding remark, $C_{i, j}$ consists of a path of length 2 or 3 in $H$ and a path $P_{i, j}$ of length 4 or 3 having only its ends in common with $H$. Since $H \cup P_{1,2} \cup P_{1,3} \cup P_{2,3}$ has radius $\leq 3$, it is planar, and from this it follows that the notation can be chosen such that $P_{1,2}: x_{2} u_{2} v_{2} y_{1}, P_{2,3}: x_{3} u_{3} v_{3} y_{2}$, and $P_{3,1}: x_{1} u_{1} v_{1} y_{3}$, where $u_{1}, v_{1}, u_{2}, v_{2}, u_{3}, v_{3}$ are all distinct. The three $x_{2}-y_{1}$ paths of $P_{1} \cup P_{2} \cup P_{1,2}$ show that $x_{2}$ and $y_{1}$ are mates. Similarly, $x_{3}$ and $y_{2}$ are mates, and $x_{1}$ and $y_{3}$ are mates. Thus we have shown that every vertex of $H$ has a mate which is in $H$. The same can be shown for $P_{1} \cup P_{2} \cup P_{1,2}$ instead of $H$. Thus $u_{2}$ has a mate $z$ in $H$. Then $z$ has two mates, a contradiction. So we can assume that no 3-path of $G$ is in two distinct 6-cycles.

We now assume that some (and hence each) 2-path is in two distinct 6-cycles. Consider now a 6-cycle $C: x_{1} x_{2} x_{3} x_{4} x_{5} x_{6} x_{1}$ as in Figure 10. The path $x_{1} x_{6} x_{5}$ is in another 6-cycle as shown in the second graph of Figure 10. Using the facts that each of the paths $x_{i} x_{i+1} x_{i+2}$ is in a 6-cycle distinct from $C$, no path of length 3 is in more than one 6-cycle, $G$ has girth 6 , and every subgraph of $G$ of radius $\leq 4$ is planar, we get the third graph of Figure 10 as a subgraph of $G$. (We draw the new paths successively noting that planarity implies that there is always only one face where the path can be drawn. We also note that we cannot have a vertex $v$ joined by three 2-paths to a 6-cycle because the three 2-paths having $v$ as a midvertex are all in two 6-cycles. This results in a nonplanar subgraph of radius 3.) The third graph of Figure 10 is a subdivision of a 3-connected graph and has only one planar embedding. The 2-path in thick lines is already contained in a 6-cycle. Another 6-cycle containing that path produces a nonplanar subgraph in $G$ of radius 4 , a contradiction.

We are left with the case that every 2-path of $G$ is contained in precisely one 6-cycle, i.e., the collection of 6-cycles in $G$ satisfies the assumption of 

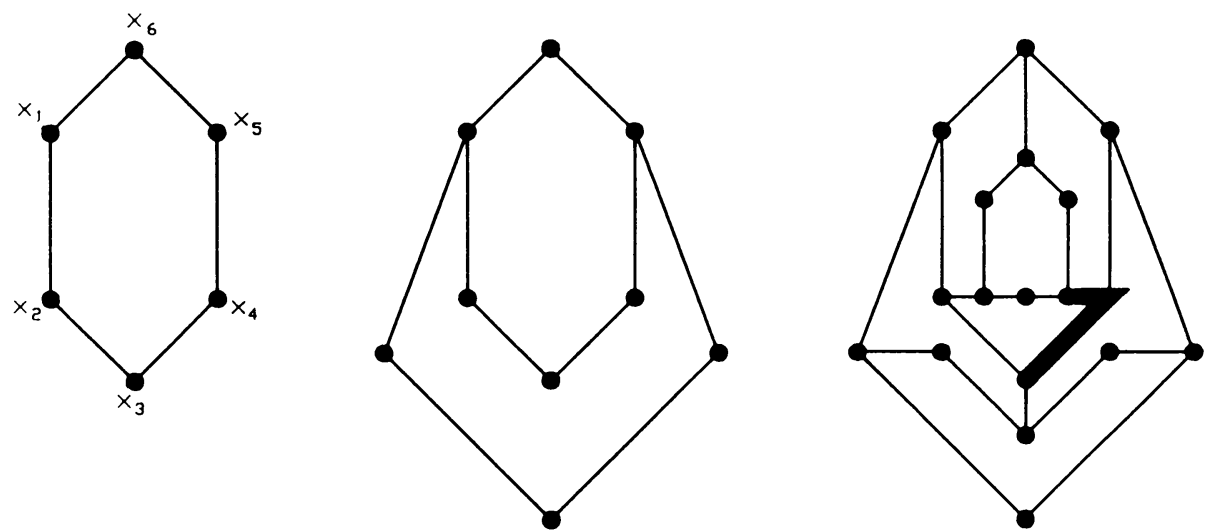

Figure 10

Theorem 3.1. Hence $G$ is one of the graphs listed in Theorem 3.1. The only edge-transitive graphs in that list are some graphs of the form $H_{k, m, r}$.

Corollary 5.2. Let $G$ be a connected cubic edge-transitive graph of order $>$ $3^{10} g(G)$. Then $G$ is the graph of the tetrahedron, or the 3-cube, or the dodecahedron, or one of the graphs $H_{k, m, r}$.

Proof. Since $n>28(g-1)$ and $G$ has minimum degree at least 3, $G$ has girth $\leq 6$, by Lemma 2.2. By the remark after Lemma $2.4, G$ has no nonplanar subgraph $H$ of radius $\leq 4$. Now Corollary 5.2 follows from Proposition 5.1 .

Proposition 5.3. If $G$ is a connected 4-regular 1-transitive graph of girth 3 and order $n>3{ }^{10} g(G)$, then $G$ is either the graph of the octahedron, or the line graph of the 3-cube, or the dodecahedron, or a 1-transitive graph of the form $H_{k, m, r}$.

Proof. For any vertex of $G, G(N(v))$ is vertex-transitive and of order 4. Hence $G(N(v))$ is a complete graph or a 4-cycle or a graph with two nonadjacent edges. In the first two cases $G$ is either a complete graph of order 5 or the graph of the octahedron. So we can assume that $v$ is contained in precisely two 3-cycles having precisely $v$ in common. Consider a 3-cycle $C: v u w v$. Since $G$ is a vertex-transitive, $G$ has 3 -cycles $C_{v}, C_{u}, C_{w}$ distinct from $C$ and containing $v, u, w$, respectively. Since no two 3-cycles incident with $v$ (and hence no two 3-cycles at all) have an edge in common, $C_{v}, C_{u}, C_{w}$ are pairwise disjoint. We now form a graph $H$ as follows: The vertices of $H$ are the 3-cycles of $G$. Two 3-cycles in $G$ are adjacent in $H$ iff they intersect in $G$. Since $G$ is 1 transitive, $H$ is 1-transitive. Clearly, $H$ is cubic and connected and has order $\frac{2}{3} n$. Also, $G$ is isomorphic to the line graph $L(H)$. So we shall prove that $H$ is either the 3-cube, or the dodecahedron, or $H$ is of the form $H_{k, m, r}$. In order to deduce this from Corollary 5.2 we shall show that

$$
\frac{2}{3} n>3^{9} g(H) \text {. }
$$


(If the graph in Corollary 5.2 is 1 -transitive we can replace $3^{10}$ by $3^{9}$.) But this follows from the assumption that $n>3^{10} g(G)$ combined with Lemma 2.5, which implies that $g(G)=g(L(H))=g(H)$.

Proposition 5.4. If $G$ is a connected 4-regular 1-transitive graph of girth $>3$ and order $n>2^{9} g(G)$, then $G$ is a graph of the form $Q_{k, m, r}$.

Proof. Assume first that $G$ contains two nonadjacent vertices $v, u$ which have the same neighbors. We shall call $v$ and $u$ mates. If a vertex has two mates, then $G$ contains a $K_{3,3}$ which is a nonplanar graph of radius 2, contrary to Lemma 2.4. So, every vertex has precisely one mate. Since a vertex is joined to two pairs of mates, it follows that $G$ is obtained from a $(n / 2)$-cycle by replacing each vertex by two nonadjacent vertices and adding all four edges between consecutive pairs. That is, $G$ is of the form $Q_{k, 1,2}$. So we can assume that $G$ has no vertices having mates.

Assume next that $G$ has two nonadjacent vertices $u, v$ with three common neighbors. So for some (and hence each) edge $e$ incident with $v$, there is a vertex $v_{e}$ joined to the three neighbors of $v$ in $G-e$. The subgraph of $G$ induced by $v$, its neighbors, and the four vertices of the form $v_{e}$ is a nonplanar graph of radius 2, contradicting Lemma 2.4. So we can assume that $G$ has no two vertices joined by three 2-paths.

Now let $G$ be embedded into $S_{g}$. By Lemma 2.3, $G$ contains a vertex $v$ which is adjacent to four faces bounded by 4-cycles

$$
\begin{array}{ll}
C_{1}: v v_{1} u_{1} v_{2} v, & C_{2}: v v_{2} u_{2} v_{3} v, \\
C_{3}: v v_{3} u_{4} v_{4} v, & C_{4}: v v_{4} u_{4} v_{1} v,
\end{array}
$$

where the indices are expressed modulo 4 . Since $G$ has no two vertices joined by three 2-paths, $u_{1}, u_{2}, u_{3}, u_{4}$ are distinct, and there is no vertex other than $v$ and $u_{i}$ which is joined to both $v_{i}$ and $v_{i+1}(i=1,2,3,4)$. If there is a vertex $z_{1}(\neq v)$ joined to $v_{1}$ and $v_{3}$, then the 1-transitivity of $G$ implies that there is a vertex $z_{2}(\neq v)$ joined to $v_{2}$ and $v_{4}$. Then the subgraph induced by $\left\{v, v_{1}, v_{2}, v_{3}, v_{4}, u_{1}, u_{2}, u_{3}, u_{4}, z_{1}, z_{2}\right\}$ is nonplanar and of radius 2 , a contradiction. So $C_{1}, C_{2}, C_{3}, C_{4}$ are the only 4-cycles containing $v$. Now Theorem 4.1 (with $\mathscr{C}$ being the collection of 4-cycles of $G$ ) implies that $G$ is of the form $Q_{k, m, r}$.

Proposition 5.5. If $G$ is a connected 5-regular 1-transitive graph of girth 3, then $G$ is $K_{6}$ or the graph of the icosahedron.

Proof. Let $v$ be any vertex of $G$, and let $H$ be the graph induced by the neighbors of $v$. Then $H$ is vertex-transitive. Since $G$ has a 3-cycle, $H$ has an edge. If $H$ is complete, then $G=K_{6}$. So assume that $H$ is not complete. Then $H$ is a 5-cycle. It follows that every edge of $G$ is in precisely two 3cycles. Now we form the dual graph $G^{\prime}$ of $G$ with respect to the 3-cycles. It is easy to see that $G^{\prime}$ is cubic, connected, 1-transitive, and of girth 5, and that 
every 2-path of $G^{\prime}$ is contained in precisely one 5-cycle. By (the proof of) Proposition 5.1, $G^{\prime}$ is the graph of the dodecahedron.

\section{VERTEX-TRANSITIVE GRAPHS OF FIXED GENUS}

In this section we prove the main theorem.

Theorem 6.1. Let $G$ be a connected vertex-transitive graph of genus $g$ and order $n>\max (90,12(g-1))$. Then $G$ is $d$-regular, where $d \leq 6$. Moreover,

(a) If $d \leq 2$, then $G$ is a cycle.

(b) If $d=3$ and $n>4 \cdot 3^{10} \mathrm{~g}$, then $G$ is a quadrilateral cylinder circuit (that is, the Cartesian product of a cycle and a $K_{2}$ ), or a quadrilateral Möbius circuit (i.e., an even length cycle with its diagonals), or $G$ is one of the graphs $H_{k, m, r}, H_{4, m, a}$, or $H_{k, m, e}$, or $G$ is obtained from a 1-transitive graph of the form $H_{k, m, r}$ (respectively $Q_{k, m, r}$ or $L\left(H_{k, m, r}\right)$ ) by applying the operation described in Figure 9(a) (respectively 9(b)).

(c) If $d=6$ and $n>6^{5} g$, then $G$ is the dual graph of one of the graphs $H_{k, m, r}, H_{4, m, a}, H_{k, d}$, or $H_{k, m, e}$.

(d) If $d=4$ and $n>2^{29} g$, then $G$ is either a graph obtained from a quadrilateral cylinder circuit by adding the two diagonals in every second 4-cycle, or $G$ is one of the graphs $Q_{k, m, r}, Q_{4, m, a}, Q_{k, m, e}$, or $G$ is obtained from an edge-transitive cubic graph of the form $H_{k, m, r}$ using the operation described in Figure $9(\mathrm{c})$ or $(\mathrm{d})$.

(e) If $d=5$ and $n>5^{7}\left(5^{7}+1\right) g$, then $G$ is obtained from one of the graphs described in Theorem 6.1(b) or (d) using the operations described in Figure 9(e), (f), (g), or $G$ is the Cartesian product of a $K_{2}$ and the square of a cycle, or $G$ is obtained from a cycle of even length by first forming the square and then adding all diagonals, or $G$ is obtained from a quadrilateral cylinder circuit by adding two diagonals in every 4-cycle.

Before we prove Theorem 6.1 we show that it implies the conjecture of Babai mentioned in the abstract. First we observe the following, by inspecting the list of graphs in Theorem 6.1:

Corollary 6.2. If $G$ is a connected vertex-transitive graph of genus $g$ and order $n \geq 10^{10} \mathrm{~g}$, then $G$ can be drawn on the torus or the Klein bottle.

In a sense, Corollary 6.2 is a more natural consequence (than Babai's conjecture) of Theorem 6.1, since the drawings in Corollary 6.2 are the "natural ones" (except for those of the graphs which can be drawn in the plane or the projective plane). In order to establish Babai's conjecture it is sufficient to show that those on the Klein bottle also have (less natural) drawings on the double torus. This is done for the graph $Q_{4,3, a}$ in [4, p. 304]. All the graphs in Corollary 6.3 below (except $H_{k, d}^{\prime}$ ) are obtained by easy modifications of the one in [4, Figure 6.25]. 
Corollary 6.3. If $G$ is a connected vertex-transitive graph of genus $g$ and order $n \geq 10^{10} g$, where $g \geq 2$ (i.e., $G$ cannot be drawn on the torus), then $G$ is one of the graphs $H_{4, m, a}, H_{4, m, a}^{\prime}, H_{k, d}^{\prime}, Q_{4, m, a}$, or $G$ is obtained from $H_{4, m, a}$ using the operation in Figure $9(\mathrm{~g})$, or from $Q_{4, m, a}$ using the operation in Figure 9(f) or from a 1-transitive graph of the form $H_{k, m, r}$ using the operation in Figure 9(a), or $G$ is obtained from a cycle of length divisible by 4 by first forming the square and then adding all diagonals.

All these graphs can be drawn on the double torus. That is, $g=2$.

The remaining part of the paper is the proof of Theorem 6.1. By Lemma $2.2, G$ is $d$-regular where $d \leq 6$. If $d \leq 2$, then $G$ is a cycle so assume that $d \geq 3$. By Lemma 2.2, $G$ has girth $\leq 6$. We consider first the cases $d=3$ and $d=6$ which are intimately related by duality and for which we use results in $\S 3$. Then we use $\S 4$ to do the case $d=4$. Finally, we dispose of the case $d=5$ in which no essentially new graphs arise. In the proof below we let $N$ denote the subgraph in $G$ induced by $N(v)$. Since $G$ is vertex-transitive, $N$ is independent of $v$. The number of edges of $N$ is the number of 3-cycles containing $v$. We color an edge of $G$ by the color $i$ if it is contained in precisely $i$ 3-cycles. Then the color of the edge $v u$ is the degree of $u$ in $N(v)$. We shall make use of the observation that every automorphism of $G$ preserves edge-colors.

The case $d=3$. We consider first the case where $G$ has girth 3 . Then $G$ is either a $K_{4}$ or $G$ is obtained from a cubic 1-transitive graph $H$ using operation (a) in Figure 9. Clearly, $g(H)=g(G)=g$. Now the proof is completed by applying Corollary 5.2 to $H$.

We consider next the case where $G$ has girth 4 . If every vertex of $G$ is in precisely one 4-cycle, then $G$ is obtained from a 1-transitive 4-regular multigraph $H$ using operation (b) in Figure 9. Since $g(H) \leq g(G)$, it follows from Propositions 5.3 and 5.4 that $H$ is of the form $Q_{k, m, r}$ or of the form $L\left(H_{k, m, r}\right)$, or $H$ is obtained from a cycle by replacing each edge by two edges. In that case $G$ is of the form $H_{k, m, r}$, where $k=2$. If some vertex (and hence each vertex) of $G$ is contained in two 4-cycles, then it is easy to see that $G$ is a quadrilateral cylinder (or Möbius) circuit. (We first show that two 4-cycles cannot have a 2-path in common. Then consider a maximal subgraph which is the Cartesian product of a 1-path and a $k$-path.)

Now we consider the case where $G$ has girth 5 . If every vertex of $G$ is in precisely one 5 -cycle, then we contract each 5 -cycle into a single vertex. This results in a 1-transitive 5-regular multigraph $H$. Since 5 is a prime, $H$ has no multiple edges. Also $g(H) \leq g(G)$. So $H$ has a $K_{3}$ by Lemma 2.3. By Proposition 5.5, this is a contradiction. So, every vertex of $G$ is contained in at least two 5-cycles. We shall show that two 5-cycles cannot have a 2-path in common. Suppose therefore (reductio ad absurdum) that $C_{1}: x_{1} x_{2} x_{3} x_{4} x_{5} x_{1}$ and $C_{2}: x_{1} x_{2} x_{3} x_{4}^{\prime} x_{5}^{\prime} x_{1}$ are distinct 5 -cycles. Clearly $x_{4}, x_{5}, x_{4}^{\prime}, x_{5}^{\prime}$ are distinct. Two vertices will be called mates if they are joined by a 2-path and two 
3-paths which are internally disjoint. Since $G$ is vertex-transitive, every vertex has a mate.

If the mate $x_{2}^{\prime}$ of $x_{2}$ is not on $C_{1} \cup C_{2}$, then the subgraph of $G$ induced by $C_{1} \cup C_{2} \cup\left\{x_{2}^{\prime}\right\}$ is nonplanar and of radius 2 , a contradiction to Lemma 2.4. So we can assume that $x_{2}^{\prime}=x_{4}$ and that $G$ has a 3-path $x_{2} x_{6} x_{7} x_{4}$. The mate of $x_{6}$ is in $C_{1} \cup C_{2}$ (because one of the three paths from $x_{6}$ to its mate begins with $\left.x_{6} x_{2}\right)$. Since $G$ has no nonplanar subgraph of radius 3 , the only possible mates for $x_{6}$ are $x_{5}$ and $x_{4}$. In each case, $G$ has a 2-path $x_{6} x_{8} x_{5}$. (Note that $x_{8} \neq x_{7}$ because $G$ has girth 5.) This shows that $x_{6}$ has two mates, namely $x_{4}$ and $x_{5}$, which are adjacent to each other but not to $x_{6}$. Hence also $x_{1}$ has a mate which is adjacent to $x_{3}$ but not to $x_{1}$. That is, one of $x_{4}, x_{4}^{\prime}$ is a mate of $x_{1}$. However, this implies the presence of a nonplanar subgraph in $G$ of radius 3 , a contradiction.

We can assume that any two 5-cycles in $G$ are either disjoint or have precisely a 1-path in common. In particular, every edge of $G$ is in at most two 5cycles. Let $E$ be the set of edges in $G$ which are in two 5-cycles. Then either $E$ is a 1-factor, or a 2-factor, or a 3-factor in $G$ because $G$ is vertextransitive. Consider two 5-cycles $C_{1}: x_{1} x_{2} x_{3} x_{4} x_{5} x_{1}$ and $C_{2}: x_{1} x_{2} y_{3} y_{4} y_{5} x_{1}$, where $x_{1} x_{2} \in E$. If there is a 5-cycle distinct from $C_{1}$ and $C_{2}$ and containing one of $x_{1} x_{5}, x_{1} y_{5}$, then it also contains the other and hence $E=E(G)$. If none of $x_{1} x_{5}, x_{1} y_{5}$ are in $E$, then $E$ is a 1 -factor in $G$. So, $E$ is not a 2 -factor. If $E \neq E(G)$, then not both $x_{4} x_{3}$ and $x_{4} x_{5}$ are in $E$. If $x_{4} x_{5} \notin E$, then the third edge incident with $x_{5}$ is in $E$ and a 5-cycle through that edge contains either $x_{1} x_{5}$ or $x_{4} x_{5}$, none of which is in $E$. This contradiction shows that $E=E(G)$. The 5-cycle distinct from $C_{1}$ through $x_{1} x_{5}$ also contains $x_{1} y_{5}$ (because no two 5-cycles have a 2-path in common). Hence every 2-path of $G$ is in precisely one 5-cycle. Now the proof of Proposition 5.1 shows that $G$ is the graph of the dodecahedron, a contradiction.

In the case $d=3$ we are left with the subcase that $G$ has girth 6 . Lemma 2.3 tells that, for every embedding of $G$ into $S_{g}$, there is a vertex $v$ which is adjacent to three faces bounded by 6 -cycles $C_{1}, C_{2}, C_{3}$. Since $G$ has girth 6, the intersection of any two of $C_{1}, C_{2}, C_{3}$ is a 2-path. So, every 2-path of $G$ is contained in a 6-cycle. If no 2-path is contained in more than one 6-cycle, then the 6-cycles of $G$ satisfy the assumption of Theorem 3.1. In that case, $G$ is a graph of form $H_{k, m, r}$, or $H_{4, m, a}$, or $H_{k, m, e}$. So assume that the 2-path in $C_{1}$ which contains precisely one edge from each of $C_{2}, C_{3}$ is in another 6-cycle $C_{1}^{\prime}$. We are going to prove that $G$ is of the form $H_{k, m, r}$ (in fact, $G$ is of the form $H_{3, m, 0}$ or $H_{3, m, 1}$ ). If two 6-cycles in $G$ have a 3-path in common, then we obtain a contradiction as in Proposition 5.1. (In the part of the proof of Proposition 5.1 where we defined mates, we only used vertex-transitivity and the property that every 2-path is in two 6-cycles. This would also hold in the present proof. For if $x_{0} x_{i} y_{i} y_{0} \quad(i=1,2,3)$ are three internally disjoint 3-paths, then an automorphism taking $x_{0}$ to $x_{1}$ shows 
the existence of a 3-path $x_{1} z_{1} z_{2} y_{i} \quad(i=2,3)$, where $z_{1}, z_{2}$ are new vertices. Now all 2-paths having $x_{1}$ as mid-vertex are in two distinct 6-cycles.) It follows that the notation can be chosen such that $C_{1}: x_{1} x_{2} x_{3} x_{4} x_{5} x_{6} x_{1}$, $C_{2}: x_{4} x_{5} x_{7} x_{8} x_{9} x_{10} x_{4}, C_{3}: x_{3} x_{4} x_{10} x_{11} x_{12} x_{13} x_{3}$, and $C_{1}^{\prime}: x_{3} x_{4} x_{5} x_{7} x_{14} x_{13} x_{3}$, where the vertices $x_{1}, x_{2}, \ldots, x_{14}$ are distinct. If the 2-path $x_{5} x_{4} x_{10}$ is contained in a 6-cycle other than $C_{2}$, then that 6-cycle would contain $x_{6} x_{5} x_{4} x_{10} x_{11}$ (since no two 6-cycles share a 3-path). But then $G$ would have a nonplanar subgraph of radius 3 , contradicting Lemma 2.4. So, every 2-path with $x_{4}$ as the mid-vertex is in precisely one 6-cycle except $x_{3} x_{4} x_{5}$ which is in precisely two 6-cycles. We shall say that $C_{1}, C_{2}, C_{3}$ are good at $x_{4}$ and that $C_{1}^{\prime}$ is bad at $x_{4}$ because $C_{1}^{\prime}$ is the unique 6-cycle through $x_{4}$ which has a 2-path in common with each other 6-cycle containing $x_{4}$. Clearly, $C_{2}$ is also good at $x_{5}$ and $x_{10}$, and $C_{1}$ is good at $x_{3}$ and $x_{5}$ (because $C_{2} \cap C_{3}, C_{2} \cap C_{1}$, and $C_{1} \cap C_{3}$ are 1-paths). Hence $C_{1}, C_{2}, C_{3}$ are good at each of their vertices because $G$ is vertex-transitive. More generally, every 6 -cycle which is good at some vertex is good at each vertex. Consequently, a 6-cycle which is bad at some vertex is bad at each of its vertices. Now, the assumption of Theorem 3.1 is satisfied with $\mathscr{C}$ being the good 6-cycles in $G$. By Theorem 3.1, $G$ is a graph of the form $H_{3, m, 0}$ or $H_{3, m, 1}$. This completes the proof of Theorem 6.1 in the case $d=3$.

The case $d=6$. By Lemma 2.3, every embedding of $G$ into $S_{g}$ has a vertex $v$ incident only with faces bounded by 3 -cycles. Let $v_{1}, v_{2}, \ldots, v_{6}$ be the neighbors of $v$ such that $C: v_{1} v_{2} \cdots v_{6} v_{1}$ is a 6-cycle. If there were another 6-cycle $C^{\prime}$ with vertex set $\left\{v_{1}, \ldots, v_{6}\right\}$, then $\bar{N}(v)$ would induce a nonplanar subgraph of radius 1 contradicting Lemma 2.4. We say that a 3-cycle $v x y v$ is good at $v$ if $x y$ is an edge of the unique 6-cycle in $G(N(v))$.

We claim that $v x y v$ is also good at $x$ and $y$. Suppose therefore that it is not good at $x$, say, where $x=v_{1}$ and $y=v_{2}$. Let $C^{\prime \prime}$ be the 6-cycle whose vertices are the neighbors of $v_{1}=x$. Then $C^{\prime \prime}$ contains $v$ and $v_{2}$ but not the edge $v v_{2}$. But then $C \cup C^{\prime \prime}$ induces a nonplanar subgraph of radius 2, a contradiction to Lemma 2.4. So, a 3-cycle is good at some vertex iff it is good at all its vertices. Now, if $\mathscr{C}^{\prime}$ denotes the collection of good 3-cycles, then the assumption of Theorem 3.2 is satisfied (with $G^{\prime}=G$ ). Hence $G$ is a dual graph of one of the graphs in Theorem 3.1. Among these, only the graphs $H_{k, m, r}^{\prime}, H_{4, m, a}^{\prime}, H_{k, d}^{\prime}$, and $H_{k, m, e}^{\prime}$ are vertex-transitive.

The case $d=4$. Assume first that $G$ has no 3-cycle. If $G$ has two nonadjacent vertices with the same neighbors, then it is easy to see that $G$ is of the form $Q_{k, 1,2}$. So assume that $G$ has no two such vertices. If $G$ is embedded into $S_{g}$, then there is a vertex $v$ which is of distance $\geq 3$ from each face which is not bounded by a 4-cycle (by Lemma 2.3). Let $C_{1}: v v_{1} u_{1} v_{2} v, C_{2}: v v_{2} u_{2} v_{3} v$, $C_{3}: v v_{3} u_{3} v_{4} v$, and $C_{4}: v v_{4} u_{4} v_{1} v$ be the facial cycles containing $v$. Since $G$ 
has no two vertices with the same neighbors and $G$ has no multiple edges, all vertices $v, v_{1}, v_{2}, v_{3}, v_{4}, u_{1}, u_{2}, u_{3}, u_{4}$ are distinct. We say that a 4cycle $C$ is good at $v$ if it contains $v$, and, for any $u, w$ which are not in $C$ but adjacent to $C, G-V(C)$ has a path of length at most 6 joining $u$ and $w$. Since all facial walks containing one of $v_{1}, v_{2}, v_{3}, v_{4}, u_{1}, u_{2}, u_{3}, u_{4}$ is a 4-cycle, each $C_{i}(1 \leq i \leq 4)$ is good at $v$. Also, there is no other 4cycle $C$ which is good at $v$. For if $C: v_{1} v v_{2} z v_{1}$ is good at $v \quad\left(z \neq u_{1}\right)$, then there is in $G-V(C)$ a path $P$ of length $\leq 6$ from $u_{1}$ to $u_{3}$, and now $P \cup C \cup C_{1} \cup C_{2} \cup C_{3} \cup C_{4}$ is a nonplanar subgraph of radius $\leq 5$, a contradiction to Lemma 2.4. Similarly, we get a contradiction if we assume there is a good 4-cycle containing $v_{1} v v_{3}$. Also note that a 4-cycle is good at some vertex iff it is good at each of its four vertices. Hence the good 4-cycles satisfy the assumption of Theorem 4.1. Therefore $G$ is one of the vertex-transitive graphs described in Theorem 4.1 (that is, $G$ is of the form $Q_{k, m, r}$, or $Q_{4, m, a}$, or $Q_{k, m, e}$ ). Now the proof is complete in the case where $G$ has girth $>3$.

We recall that $N=G(N(v))$ for each vertex $v$ of $G$. In the case $d=4$ we are left with the case that $N$ has at least one edge. We now consider the case where $N$ has precisely one edge. Then the 3-cycles in $G$ form a 2factor. Let $G^{\prime}$ be obtained from $G$ by contracting each 3-cycle into a vertex. Then $G^{\prime}$ is a 6-regular vertex-transitive multigraph. If each vertex of $G^{\prime}$ has only two neighbors, then $G$ is of the form $Q_{k, 2,0}$. If each vertex of $G^{\prime}$ has three neighbors, then the underlying graph of $G^{\prime}$ is a 1-transitive cubic graph, and $G$ is obtained from that by using the operation (d) in Figure 9. Since $g\left(G^{\prime}\right) \leq g(G)$, the proof is now completed using Lemma 2.2 and Proposition 5.1. (Since $G$ has no nonplanar subgraph of radius $\leq 5$, the operation (d) must preserve the local planarity as indicated in Figure $9(\mathrm{~d})$.) If $G^{\prime}$ is a 6-regular graph, then $G^{\prime}$ is one of the graphs $H_{k, m, r}^{\prime}, H_{4, m, a}^{\prime}, H_{k, d}^{\prime}$, or $H_{k, m, e}^{\prime}$ by the previous case $d=6$. So $G$ is obtained from one of these graphs by blowing each vertex up into a 3-cycle such that the local planarity is preserved, every vertex is in precisely one 3 -cycle, and $G$ is vertex-transitive. We leave it to the reader to verify that this is not possible.

Consider next the case where $d=4$ and $N$ has precisely two edges incident with the same vertex. Then each vertex is incident with precisely one edge which is not in a 3-cycle. If we delete all edges not in 3-cycles, then the resulting graph is cubic vertex-transitive and each of its vertices is in precisely two 3-cycles. But, such a graph does not exist.

We consider now the case where $N$ has precisely two edges which are not incident with the same vertex. Hence every vertex is in precisely two 3-cycles, and it is easy to see that every 3-cycle intersects precisely three other 3-cycles which are pairwise disjoint. Hence $G$ is the line graph of a cubic graph $G^{\prime}$. That is, $G$ is obtained from $G^{\prime}$ using the operation (c) in Figure 9. Since $G$ is vertex-transitive, $G^{\prime}$ is edge-transitive. By Lemma 2.5, $g\left(G^{\prime}\right)=g(G)=g$. By Corollary 5.2, $G^{\prime}$ is of the form $H_{k, m, r}$. 
We claim that $N$ cannot have a vertex of degree 3 (when $d=4$ ). For if this were the case, then each vertex $v$ in $G$ would have a neighbor $v^{\prime}$ such that $v$ and $v^{\prime}$ have the same neighbors outside $\left\{v, v^{\prime}\right\}$. Each of the common three neighbors $u_{i}(i=1,2,3)$ of $v$ and $v^{\prime}$ has the same property, i.e., there is a neighbor $u_{i}^{\prime}$ of $u_{i}$ such that $u_{i}$ and $u_{i}^{\prime}$ have the same neighbors outside $\left\{u_{i}, u_{i}^{\prime}\right\}$. Clearly, $u_{i}^{\prime} \in\left\{v, v^{\prime}, u_{1}, u_{2}, u_{3}\right\}$. This can only be satisfied if $G=K_{5}$, a contradiction. So $N$ has maximum degree 2, that is, $N$ is either a 3-path or a 3-cycle (and an isolated vertex) or a 4-cycle in the remaining cases where $d=4$.

If $N$ is a 3-cycle (and an isolated vertex), then the contraction of each $K_{4}$ in $G$ results in a 1-transitive 4-regular multigraph $G^{\prime}$. If $G^{\prime}$ is a cycle, then $G$ is obtained from a quadrilateral cylinder circuit by adding two diagonals to every second 4-cycle. So assume that $G^{\prime}$ has no multiple edges. Since $g\left(G^{\prime}\right) \leq g(G)$, Propositions 5.3 and 5.4 imply that $G^{\prime}$ is one of the graphs $L\left(H_{k, m, r}\right)$ or $Q_{k, m, r}$. Then the two 6-cycles containing a fixed edge in $H_{k, m, r}$ or the four 4-cycles in $Q_{k, m, r}$ containing any fixed vertex correspond in $G$ to a nonplanar graph of radius $\leq 7$, a contradiction to Lemma 2.4 .

If $N$ is a 4-cycle, then every vertex which is a neighbor of a vertex $v$ is joined to precisely one nonneighbor of $v$. That nonneighbor of $v$ has to be the same for all neighbors of $v$ since they are all the center of a 4-wheel. Hence $G$ is the graph of the octahedron, a contradiction.

In the case $d=4$ we are left with the case where $N=G(N(v))$ is a 3-path: $v_{1} v_{2} v_{3} v_{4}$. We shall show that $G$ is the square of a cycle (i.e., $G$ is of the form $\left.Q_{k, 1,1}\right)$ and we shall not make use of the fact that $n$ is large. Recall that an edge has color $i$ if it is contained in precisely $i$ 3-cycles. Thus $v v_{1}$ and $v v_{4}$ have color 1 while $v v_{2}$ and $v v_{3}$ have color 2 .

Every automorphism of $G$ preserves edge-colors. Consider the vertex $v_{1}$. The 3-path induced by $N\left(v_{1}\right)$ is of the form $v v_{2} z_{1} z_{2}$. In particular, $v_{1} v_{2}$ has color 2. Similarly, $v_{3} v_{4}$ has color 2 . So, every edge of color 1 is contained in a 3 -cycle whose other edges have color 2 . Hence the subgraph of $G$ consisting of edges of color 2 is connected and 2-regular, that is, the edges of color 2 form a Hamiltonian cycle $C$. The 2-path of $C$ whose mid-vertex is $v$ is contained in a 3-cycle in $G$. Hence every 2-path of $C$ is contained in a 3-cycle of $G$ which implies that $G$ contains the square of $C$. Since $G$ is 4-regular, $G$ is the square of $C$. We have now completed the proof when $d=4$.

The case $d=5$. By Lemma 2.3, $N$ has a path system with at least three edges. We consider first the case where $N$ has a vertex of degree 4 , that is, for each vertex $v$ there is a vertex $v^{\prime}$ in $N(v)$ such that $N(v) \backslash\left\{v^{\prime}\right\}=N\left(v^{\prime}\right) \backslash\{v\}$. We say that $v$ and $v^{\prime}$ are mates. A vertex cannot have two mates because $G(\bar{N}(v))$ is planar. So, if we contract each edge joining two mates into a single vertex, then we obtain a multigraph whose underlying graph is a cycle. Hence $G$ is obtained from a quadrilateral cylinder circuit by adding all diagonals to all 4-cycles. In what follows we therefore assume that $N$ contains a path system 
with three edges (by Lemma $2.3(\mathrm{~b})$ ) and has maximum degree at most 3 . Now we prove:

(1) $N$ has either none or at least two vertices of degree 3 .

Proof of (1). Suppose (reductio ad absurdum) that $N=G(N(v)$ ) has precisely one vertex, say $u$, of $N$-degree 3 . Then the edges of color 3 form a 1 -factor. If we delete the edges of color 3 , we obtain a connected vertex-transitive 4-regular graph $G^{\prime}$ of girth 3 and genus $g\left(G^{\prime}\right) \leq g(G)=g$. Then $G^{\prime}$ is one of the graphs found in the case $d=4$. Also, for every vertex $v$ in $G^{\prime}$, there is a vertex $u$ such that $v$ and $u$ are joined by three 2-paths. However, no graph of girth 3 found in the case $d=4$ has this property. This contradiction proves (1).

(2) Either $N$ has none or at least two 3-cycles.

Proof of (2). Suppose (reductio ad absurdum) that $N$ has precisely one 3-cycle $v_{1} v_{2} v_{3} v_{1}$. Then every vertex of $G$ is contained in precisely one $K_{4}$. Let $G^{\prime}$ be obtained from $G$ by contracting each $K_{4}$ into a single vertex. Then $G^{\prime}$ is 8-regular, vertex-transitive, and of genus $\leq g$. The automorphism group of $G^{\prime}$ partitions $E\left(G^{\prime}\right)$ into at most two orbits. Hence $G^{\prime}$ has at most two edge-multiplicities. (The multiplicity of a multiple edge between $x$ and $y$ is the number of edges joining $x$ and $y$.) These multiplicities must be 1, 2, or 4 . Two vertices in $G^{\prime}$ cannot be joined by four edges because that would imply the existence of a nonplanar subgraph in $G$ of radius 2 or two 3-cycles in $N$. So all edge-multiplicities are 1 or 2 . By Lemma $2.2, G^{\prime}$ has multiple edges. If $G^{\prime}$ also has single edges, then $E\left(G^{\prime}\right)$ has two orbits with four edges (incident with $v$ ) in each. In this case the underlying graph $H$ of $G^{\prime}$ is 6-regular. So we can assume that $H$ is 4-regular or 6-regular. From the cases $d=4,6$ we know the structure of $G^{\prime}$. In any case, if $v^{\prime}$ is a vertex of $G^{\prime}$, then $G^{\prime}$ has a cycle of length at most 10 (or a 4-path) containing all neighbors of $v^{\prime}$. (The number 10 occurs when $H$ is the line graph of a cubic graph of girth 6.) In $G$ this produces a nonplanar graph (namely a graph that can be contracted into $K_{5}$ ) of radius $\leq 7$, a contradiction to Lemma 2.4.

(3) $N$ has no 3-cycle.

Proof of (3). Suppose (reductio ad absurdum) that $v_{1} v_{2} v_{3} v_{1}$ is a 3-cycle in $N$. By (2), $N$ has another 3-cycle as well. Since $N$ has no vertex of degree 4, that 3 -cycle must be of the form $v_{1} v_{2} v_{4} v_{1}$. The fifth vertex $v_{5}$ in $N$ cannot be joined to $v_{1}$ or $v_{2}$ because $N$ has no vertex of degree 4 . Also, $v_{5}$ cannot be joined to both $v_{3}$ and $v_{4}$ because $G(\bar{N}(v))$ then would be a nonplanar graph of radius 1 . So $v_{5}$ has $N$-degree 0 or 1 . Then $v v_{5}$ is the only edge incident with $v$ of color $<2$. If we delete all edges of color $<2$, then the resulting graph is a 4-regular vertex-transitive graph in which each neighborhood graph is a $K_{4}$ minus an edge. It is easy to see that no such graph exists. This contradiction proves (3). 
(4) $N$ has no vertex of degree 3.

Proof of (4). Suppose (reductio ad absurdum) that $v_{1}$ has $N$-degree 3. By (1), $N$ has another vertex $v_{2}$ of degree 3. By (3), $v_{1}$ and $v_{2}$ are nonadjacent and have the same three neighbors in $N$. But then $G(\bar{N}(v))$ is nonplanar and of radius 1 . This contradiction proves (4).

(5) $N$ is not a 5-cycle.

Proof of (5). Suppose (reductio ad absurdum) that $N$ is a 5-cycle. Then the collection of 3-cycles define an embedding of $G$ in some surface. Since that embedding is a triangulation, $G$ has at least $3 n-6$ edges by a remark before Lemma 2.2. On the other hand, $G$ has $\frac{5}{2} n$ edges. Hence $n \leq 12$, a contradiction which proves (5).

By (4) and (5), $N$ is either a 3-path (and an isolated vertex), or a 4-path, or a 4-cycle (and an isolated vertex), or a path system consisting of a 1-path and a 2-path. We consider first the case where $N$ has three edges forming the disjoint paths $v_{1} v_{2} v_{3}$ and $v_{4} v_{5}$. The vertex $v$ is incident with precisely one edge (namely $v v_{2}$ ) of color 2 . Thus also $v_{2}$ is only incident with one edge of color 2. In particular, $v_{1} v_{2}$ and $v_{2} v_{3}$ have color 1 . So, if we delete from $G$ all edges of color 2 , then the resulting graph $G^{\prime}$ is connected 4-regular and vertex-transitive. Hence $G^{\prime}$ is one of the graphs described in the case $d=4$. $G$ is obtained from $G^{\prime}$ by adding a 1 -factor consisting of edges each of which is a diagonal in a 4-cycle of $G^{\prime}$. Moreover, every vertex of $G^{\prime}$ is in at most one 3-cycle, so $G^{\prime}$ is not one of the graphs indicated in Figure 9(c). We have previously determined the structure of $G^{\prime}$. We now consider each of the graphs that $G^{\prime}$ may be isomorphic to. It then follows that $G$ has the structure indicated in Figure 9(f). (Note that $G^{\prime}$ cannot be the 4-regular graph shown in Figure 9(d) because adding a diagonal in a 4-cycle would create a 3-path in $N$. Also note that in order to obtain $G$ from $G^{\prime}$ we never add diagonals to two 4-cycles that have an edge in common because $N$ has no 3-path.)

Assume next that $N$ is a 3-path $v_{1} v_{2} v_{3} v_{4}$ and an isolated vertex $v_{5}$. A 3-cycle containing $v$ has at most one edge incident with $v$ of color 1 . The same holds for 3-cycles incident with $v_{1}$. Hence $v_{1} v_{2}$ has color 2 . Similarly, $v_{4} v_{3}$ has color 2 . It follows that the graph $G^{\prime}$ obtained from $G$ by deleting all edges of color 1 is connected. Moreover, $G^{\prime}$ is cubic and vertex-transitive and of genus $\leq g$. So, $G^{\prime}$ is one of the graphs described in the case $d=3$. Since $v$ is incident with precisely two edges of color 2 , the edges of color 2 form a 2 -factor in $G^{\prime}$, and the edges of color 1 are precisely those which join pairs of vertices which are connected by a 2-path in the 2-factor consisting of color 2 edges. In particular, $v_{2} v_{3}$ has color 1 and hence $G^{\prime}$ has no 3-cycle. If $G^{\prime}$ has girth 4 , then $G^{\prime}$ is a quadrilateral cylinder (or Möbius) circuit or else $G^{\prime}$ is one of the graphs indicated in Figure 9(b). In the former case the only possible 2 -factor in $G^{\prime}$ are the peripheral cycles (or cycle) since $N$ has no 3 -cycle. Hence $G$ is the Cartesian product of a $K_{2}$ and the square of a cycle, or 
$G$ is obtained from a cycle by forming the square and then adding all diagonals. In the latter case we first observe that the 2-factor consisting of color 2 edges contains no three edges of a 4-cycle. So it contains two nonadjacent edges of each 4-cycle and also all four edges going out from the 4-cycle. It is then easy to find a nonplanar subgraph of radius $\leq 7$ in $G$, a contradiction. If $G^{\prime}$ has girth $>4$, then $G^{\prime}$ is a hexagonal tiling of the torus or the Klein bottle, by the case $d=3$. The 2 -factor consisting of color 2 edges cannot contain a 3-path in a facial cycle (because $G$ would then contain a nonplanar graph of radius 2, contradicting Lemma 2.4). Hence $G$ has the structure indicated in Figure $9(\mathrm{~g})$, where the edges of color 1 are indicated in dotted lines.

We now consider the case where $N$ is a 4-path $v_{1} v_{2} v_{3} v_{4} v_{5}$. Precisely two edges incident with $v$ (namely $v v_{1}$ and $v v_{5}$ ) have color 1 while the other three have color 2. Let $v v_{2} u_{1} u_{2} u_{3}$ be the 4-path in $G\left(N\left(v_{1}\right)\right)$. It follows that $v_{1} v_{2}, v_{1} u_{1}, v_{1} u_{2}$ have color 2. A similar argument shows that $v_{5} v_{4}$ has color 2. The 4-path in $G\left(N\left(v_{2}\right)\right)$ contains the 3-path $u_{1} v_{1} v v_{3}$. It follows that one of $v_{2} u_{1}, v_{2} v_{3}$ has color 1 and the other has color 2. So, one of $v, v_{1}$ (and hence both of them) is contained in a 3-cycle whose edges have color 2. Let $G^{\prime}$ be the graph consisting of all edges of color 2. Then $G^{\prime}$ is cubic, connected, vertextransitive, and every vertex is in a 3 -cycle. Hence $G^{\prime}$ is obtained from a 1 transitive graph $G^{\prime \prime}$ using operation (a) in Figure 9. Since $g\left(G^{\prime \prime}\right)=g\left(G^{\prime}\right) \leq g$, Proposition 5.1 implies that $G^{\prime \prime}$ is a 1-transitive graph of the form $H_{k, m, r}$. $G$ is obtained from $G^{\prime}$ by adding a 2-factor such that all edges in $G-E\left(G^{\prime}\right)$ join vertices of distance 2 in $G^{\prime}$. Now $G^{\prime}$ has the local structure shown in Figure 11. Since $G$ has no small nonplanar graph and is vertex-transitive, the edges of $G-E\left(G^{\prime}\right)$ can only be added as indicated by curved lines in Figure 11. (Since there are six color 1 edges incident with each 3-cycle in $G^{\prime}$, any two adjacent 3 -cycles in $G^{\prime}$ are joined by two color 1 edges.) The resulting graph is obtained from $G^{\prime \prime}$ by operation (e) in Figure 9 .

We finally assume that $N$ is a 4-cycle $v_{1} v_{2} v_{3} v_{4} v_{1}$ and an isolated vertex $v_{5}$. Let $G^{\prime}$ be obtained from $G$ by deleting all edges of color 0 . The resulting graph is a 4-regular graph in which each neighborhood graph is a 4-cycle. Then each component of $G^{\prime}$ is the graph of the octahedron. Contracting each component of $G^{\prime}$ into a single vertex results in a 6-regular 1-transitive multigraph $G^{\prime \prime}$. The underlying graph of $G^{\prime \prime}$ is either (by the cases $d=3,6$ ) a graph of the form $H_{k, m, r}$ or $H_{k, m, r}^{\prime}$, or $G^{\prime \prime}$ is a cycle. If $G^{\prime \prime}$ is of the form $H_{k, m, r}$ or $H_{k, m, r}^{\prime}$, then $G$ has a nonplanar subgraph of radius $\leq 7$, a contradiction to Lemma 2.4. So $G^{\prime \prime}$ is a cycle. The graph of the octahedron is a $K_{6}$ minus a 1 -factor. Each octahedron in $G$ is joined by three edges to each neighboring octahedron. Since $G$ is vertex-transitive, the subgraph induced by the three vertices in the octahedron which are joined to one of the neighboring octahedra is a $K_{3}$. This describes $G$ completely. $G$ is the graph obtained from $H_{k, m, r}$ (with $k=3$ and $r=0$ ) as indicated in Figure $9(\mathrm{~g})$.

This finally completes the proof of Theorem 6.1 . 


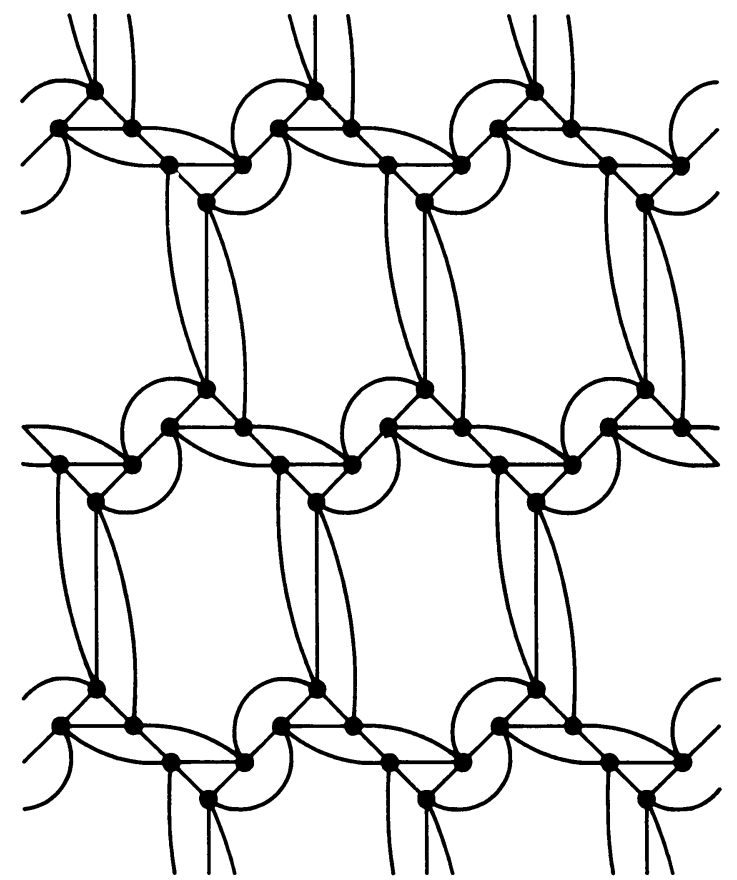

FIGURE 11

\section{REFERENCES}

1. A. Altschuler, Construction and enumeration of regular maps on the torus, Discrete Math. 4 (1973), 201-217.

2. J. Battle, F. Harary, Y. Kodama, and J. W. T. Youngs, Additivity of the genus of a graph, Bull. Amer. Math. Soc. 68 (1962), 565-568.

3. H. Fleischner and W. Imrich, Transitive planar graphs, Math. Slovaca 29 (1979), 97-106.

4. J. L. Gross and T. W. Tucker, Topological graph theory, Wiley, New York, 1987.

5. B. Grünbaum and G. C. Shephard, Tilings and patterns, Freeman, New York, 1987.

6. S. Stahl and L. W. Beineke, Blocks and the nonorientable genus of a graph, J. Graph Theory 1 (1977), 75-78.

7. C. Thomassen, Embeddings of graphs with no short noncontractible cycles, J. Combin. Theory Ser. B 48 (1990), 155-177.

8. __ Embeddings and minors, in Handbook of Combinatorics (M. Grötschel, L. Lovász, and R. L. Graham, eds.), North-Holland (to appear).

9. __ The graph genus problem is NP-complete, J. Algorithms 10 (1989), 568-576.

10. _ The Jordan-Schönflies theorem and the classification of surfaces, Amer. Math. Monthly (to appear).

11. T. W. Tucker, Finite groups acting on surfaces and the genus of a group, J. Combin. Theory Ser. B 34 (1983), 82-98.

12. W. T. Tutte, Connectivity in graphs, Univ. of Toronto Press, Toronto, 1966.

13. L. Babai, Vertex-transitive graphs and vertex-transitive maps, J. Graph Theory (to appear).

Mathematical Institute, The Technical University of Denmark, Building 303, DK2800 LyNGBY, DeNMARK 